\title{
Evaluation of the arbitrary Langrangian-Eulerian vertical coordinate method in the MPAS-Ocean Model
}

\author{
Mark Petersen ${ }^{\mathrm{a}, *}$, Doug W. Jacobsen ${ }^{\mathrm{b}}$, Todd D. Ringler ${ }^{\mathrm{b}}$, Matthew W. \\ Hecht $^{\mathrm{a}}$, Matthew E. Maltrud ${ }^{\mathrm{b}}$ \\ ${ }^{a}$ Computer and Computational Science Division, Los Alamos National Laboratory, \\ Los Alamos, New Mexico 87545, USA \\ ${ }^{b}$ Theoretical Division, Los Alamos National Laboratory, \\ Los Alamos, New Mexico 87545, USA
}

\begin{abstract}
The vertical coordinate of the Model for Prediction Across Scales-Ocean (MPAS-Ocean) uses the Arbitrary Lagrangian-Eulerian (ALE) method, which offers a variety of configurations. When fully Eulerian, the vertical coordinate is fixed like a z-level ocean model; when fully Lagrangian there is no vertical transport through the interfaces so that the mesh moves with the fluid; additional options for vertical coordinates exist between these two extremes, including z-star, z-tilde, sigma, and isopycnal coordinates. Here we evaluate spurious diapycnal mixing in MPAS-Ocean in several idealized test cases as well as real-world domains with full bathymetry. Mixing data is compared to several other ocean models, including the Parallel Ocean Program (POP) z-level and z-star formulations. In three-dimensional domains, MPAS-Ocean has lower spurious mixing that other ocean models. A series of simulations show that this is likely due to MPAS-Ocean's hexagon-type horizontal grid cells combined with a flux-corrected transport tracer advection scheme designed for these unstructured meshes.

The frequency-filtered vertical coordinate of Leclair and Madec (2011) (also called z-tilde) has been implemented and analyzed in MPAS-Ocean. This addition allows low-frequency vertical transport to pass through the vertical interface in an Eulerian manner, while high-frequency vertical oscillations, such as internal gravity waves, are treated in a Lagrangian manner.
\end{abstract}

\footnotetext{
* Corresponding author

Email address: mpetersen@lanl.gov (Mark Petersen)
} 
Z-tilde leads to a substantial reduction in vertical transport across layer interfaces, and a reduction in spurious diapycnal mixing.

Keywords: ocean modeling, diapycnal ocean mixing, Arbitrary Lagrangian-Eulerian coordinate method, unstructured mesh

\section{Introduction}

The choice of vertical coordinate is intrinsic to the character of a global ocean model. Historically, ocean models have been classified into groups according to their vertical coordinate. Z-level models have fixed, level layer interfaces (Griffies et al., 2005; Smith et al., 2010). Z-level models are straightforward to implement and have no pressure gradient errors due to tilted layers, but are prone to high spurious diapycnal mixing (Willebrand, 2001; Legg et al., 2006). Isopycnal models substantially reduce diapycnal mixing because adiabatic motion is treated entirely in a Lagrangian manner, thus producing no spurious mixing. Purely isopycnal models have insufficient resolution in the mixed layer and other neutrally-stratified regions (Bleck, 1978). Hybrid coordinate models combine the advantages of z-level in the upper ocean for higher resolution and isopycnal coordinates in the deep ocean to reduce diapycnal mixing (Bleck, 2002). Sigma coordinates are terrain-following so that the full vertical resolution may be used throughout, and are particularly useful for coastal modeling (Shchepetkin and McWilliams, 2005). Special care must be taken to avoid errors in the pressure gradient when layers have large slopes along steep topography in sigma coordinate models (Shchepetkin and McWilliams, 2003, 2011).

Amongst z-level models, several improvements have been introduced that are now standard in this class of models. Partial bottom cells improves the representation of bathymetry, and thus improves advection and mixing processes along the ocean floor (Adcroft et al., 1997; Pacanowski and Gnanadesikan, 1998). Scaling the vertical coordinate in proportion to the sea surface height ( $\mathrm{SSH}$ ), sometimes called z-star, reduces spurious mixing due to surface gravity waves and allows the upper layers to be very thin (Adcroft and Campin, 2004). Without this improvement, z-level models must include all SSH variations in the top layer, so it must be at least several meters thick, or use a rigid lid (Smith et al., 1992).

A new addition to improvements in z-level models is the frequency-filtered vertical coordinate of Leclair and Madec (2011), named z-tilde. This scheme 
applies a time-frequency filter to the horizontal divergence in each grid cell. High-frequency oscillations that occur on a time scale of less than several days, such as internal gravity waves, are filtered out of the vertical transport, so that the vertical grid moves with these waves in a Lagrangian fashion. The remaining low-frequency motions, such as seasonal oscillations and advection of large water masses, move through the vertical interfaces as they do in a typical z-level model. Because much of the spurious mixing is caused by highfrequency oscillations, this scheme offers the advantage of reduced diapycnal mixing intrinsic to isopycnal models, but avoids the complexity of remapping schemes, choosing target grids, and supporting massless layers associated with hybrid-isopycnal models. The end result is a vertical coordinate that may conveniently use all the machinery of a z-level model, such as topography and initial conditions, but where the vertical grid may follow small, fast oscillations in a Lagrangian manner to reduce spurious mixing.

In the Arbitrary Lagrangian-Eulerian (ALE) method, the grid is remapped so that cell interfaces may remain at a fixed location (Eulerian), may move with the fluid (Lagrangian), or anything in between (Hirt et al., 1974). It may be configured for any of the vertical coordinate types discussed above. ALE was developed to take advantage of the properties of Lagrangian algorithms, where the model mesh naturally tracks the material's properties, but to avoid the resulting distortions in the computational domain (Donea et al., 2004). ALE methods were applied to general vertical coordinates in ocean models by Bleck (1978).

In a typical ALE algorithm, the mesh may be specified in an arbitrary manner at regular intervals in time, and variables are subsequently remapped to that new mesh. The Model for Prediction Across Scales-Ocean (MPASOcean) implementation determines a desired thickness at each time step based on a number of criteria, and then computes the vertical transport through the layer interface that is required to acquire the desired thickness. Rather than explicitly remapping variables to the new grids, the ocean model simply transports water volume and tracers using this vertical transport in the normal prognostic conservation equations (the mass equation is written as a volume or thickness equation for Boussinesq fluids). This is the Lagrangian treatment of the vertical direction described in Adcroft and Hallberg (2006). The method ensures that water volume and tracers are conserved to machine precision.

Given the advantages discussed above, we have chosen an ALE framework to model vertical transport within MPAS-Ocean. Vetted configurations 
of MPAS-Ocean employing a vertical ALE treatment include z-level, z-star (Ringler et al., 2013), idealized isopycnal (Ringler and Gent, 2011), as well as z-tilde, partial bottom cells, and sigma coordinates (all in this paper). All these have been tested in realistic high resolution global domains except sigma and isopycnal coordinates, which have been tested in idealized domains to date.

MPAS-Ocean is an open-source, global ocean model (see http://mpasdev.github.io). MPAS-Ocean is one component of a family of climate models within the MPAS framework, including atmosphere (Skamarock et al., 2012), sea-ice, and land-ice models. MPAS components use horizontally unstructured meshes based on Voronoi tessellations, which allow high-resolution regions within a low-resolution global mesh, with a smoothly varying mesh transition between them. The motivation for this design is to provide a single ocean modeling system that can be used with traditional quasi-uniform meshes as well as regional configurations to investigate regional effects of climate change. All simulations presented here use MPAS-Ocean version 2.0, released in November 2013.

The purpose of this paper is two-fold: (1) to verify the behavior of MPASOcean against published results for numerous idealized test cases and realworld domains; and (2) to demonstrate the advantages of z-tilde in reducing spurious vertical mixing. Ilicak et al. (2012) proposed four idealized test cases that are repeated here, and provided comparison data for Modular Ocean Model (MOM), MIT General Circulation Model (MITgcm), Regional Ocean Modeling System (ROMS), and the Generalized Ocean Layered Model (GOLD). To that we have added data from MPAS-Ocean run in zlevel, z-star, isopycnal, sigma, and z-tilde coordinates, as well as POP, a quadrilateral-grid ocean model developed at Los Alamos National Laboratory, using z-level and z-star coordinates. The test cases include the (1) lock exchange, (2) overflow, (3) internal wave, (4) baroclinic eddies, and (5) real world. These cases progress from simple domains and initial conditions to more complex dynamics and topography, so that spurious mixing may be measured under a variety of conditions.

The design of the MPAS unstructured horizontal mesh has already been described in several publications (Ringler et al., 2008; Thuburn et al., 2009; Ringler et al., 2010), while high-resolution and variable-resolution global simulations were verified against observations in Ringler et al. (2013). The current work may be considered a follow-on to Ringler et al. (2013), where the prior is a detailed vetting of the horizontal infrastructure of MPAS-Ocean, 
while the current does the same for the vertical.

This paper is organized as follows. Section 2 details the algorithms of the vertical grid, with subsections on the overall ALE formulation and z-tilde. Section 3 presents the results from four idealized test cases, while section 4 proceeds to realistic simulations of global oceans with full bathymetry. Finally, the work is summarized in the concluding Section 5.

\section{Numerical Algorithm}

This section describes the equations and discretization of the MPASOcean vertical coordinate. All horizontal variables and operations are continuous to simplify the presentation. The horizontal discretization is discussed in Sections 2, 3.1, and A.4 of Ringler et al. (2013), and further details may be found in the MPAS-Ocean User's Guide (Jacobsen et al., 2013). All functionality described here is available in MPAS-Ocean version 2.0.

The MPAS-Ocean governing equations are for a hydrostatic, incompressible fluid using the Boussinesq approximation. Conservation of momentum, volume, and tracers are written for layer $k$ as

$$
\begin{aligned}
& \left.\frac{\partial u_{k}}{\partial t}+q_{k} h_{k} u_{k}^{\perp}+\overline{\left[w_{:}^{t} \delta z^{t}\left(u_{:}\right)\right.}\right]_{k}^{m} \\
& \quad=-\frac{1}{\rho_{0}} \nabla p_{k}-\frac{\rho_{k} g}{\rho_{0}} \nabla z_{k}^{m i d}-\nabla K_{k}+\left[\mathbf{D}_{h}^{u}\right]_{k}+\left[\mathbf{D}_{v}^{u}\right]_{k}+\mathcal{F}_{k}^{u} \\
& \frac{\partial h_{k}}{\partial t}+\nabla \cdot\left(h_{k} \mathbf{u}_{k}\right)+w_{k}^{t}-w_{k+1}^{t}=0, \\
& \frac{\partial\left(h_{k} \varphi_{k}\right)}{\partial t}+\nabla \cdot\left(h_{k} \mathbf{u}_{k} \varphi_{k}\right)+\bar{\varphi}_{k}^{t} w_{k}^{t}-\bar{\varphi}_{k+1}^{t} w_{k+1}^{t}=\left[\mathbf{D}_{h}^{\phi}\right]_{k}+\left[\mathbf{D}_{v}^{\phi}\right]_{k}+\mathcal{F}_{k}^{\phi}
\end{aligned}
$$

where all symbols are defined in Tables 1 and 2. Additional equations diagnose the pressure from hydrostatic balance and the density from an equation of state, as described in section A.1 of Ringler et al. (2013). Overbars indicate that variables are vertically averaged to the top $(t)$ or middle $(m)$ of the layer, and $\delta z^{t}$ is a first-order derivative in the vertical. The term $\nabla z_{k}^{\text {mid }}$ does not appear in z-level models, but is required to compensate for pressure gradients due to the tilting of layers (Adcroft and Campin, 2004, Appendix A).

\subsection{ALE Algorithm}

The ALE algorithm in the MPAS-Ocean code provides the vertical transport of fluid through the top cell interface, $w^{t}$, which is then used in the 
Table 1: Latin variables used in prognostic equation set. All variables are located at the center of the layer in the vertical unless noted.

\begin{tabular}{ccc}
\hline \hline symbol & name & notes \\
\hline $\mathbf{D}_{h}^{u}, \mathbf{D}_{v}^{u}$ & mom. diffusion terms & $h$ horizontal, $v$ vertical \\
$D_{h}^{\varphi}, D_{v}^{\varphi}$ & tracer diffusion terms & \\
$f$ & Coriolis parameter & \\
$\mathcal{F}^{u}$ & momentum forcing & \\
$\mathcal{F}^{\varphi}$ & tracer forcing & constant \\
$g$ & grav. acceleration & \\
$h$ & layer thickness & $K=|\mathbf{u}|^{2} / 2$ \\
$\mathbf{k}$ & vertical unit vector \\
$K$ & kinetic energy & $q=\eta / h$ \\
$p$ & pressure & a tracer $\varphi$ \\
$q$ & potential vorticity & - \\
$S$ & salinity & time \\
$t$ & horizontal velocity & normal component to edge \\
$u$ & horizontal velocity & tangential component to edge \\
$u^{\perp}$ & horizontal velocity & - \\
$\mathbf{u}$ & vertical transport & top of layer in vertical \\
$w^{t}$ & vertical coordinate & positive upward \\
$z$ & z-location of middle of layer & \\
$z^{m i d}$ & &
\end{tabular}

time-integration of the thickness, momentum and tracer equations. The ALE vertical grid moves in time; the cell interface locations may be determined by summing layer thicknesses $h_{k}$ from the bottom bathymetry up.

A summary of the ALE algorithm is as follows, with variables defined in Table 4. This is executed within each time step, where all variables at the previous time step, $n$, are known.

1. Compute desired thickness for the new time,

$$
h_{k}^{A L E}=h_{k}^{r e s t}+h_{k}^{S S H}+h_{k}^{h f}+h_{k}^{\text {limit }}
$$

2. Solve for vertical transport $w^{t}$ from (2),

$$
w_{k}^{t}=w_{k+1}^{t}-D_{k}-\frac{h_{k}^{A L E}-h_{k}^{n}}{\Delta t}
$$


Table 2: Greek variables used in prognostic equation set. All variables are located at the center of the layer in the vertical.

\begin{tabular}{ccc}
\hline \hline symbol & name & notes \\
\hline$\omega$ & relative vorticity & $\omega=\mathbf{k} \cdot(\nabla \times \mathbf{u})$ \\
$\eta$ & absolute vorticity & $\eta=\omega+f$ \\
$\Theta$ & potential temperature & a tracer $\varphi$ \\
$\kappa_{h}, \kappa_{v}$ & tracer diffusion & horizontal and vertical \\
$\nu_{h}, \nu_{v}$ & viscosity & horizontal and vertical \\
$\rho$ & density & \\
$\rho_{0}$ & reference density & constant \\
$\varphi$ & generic tracer & e.g. $\Theta, S$ \\
\hline
\end{tabular}

3. Solve for new thickness, $h_{k}^{n+1}$, using the continuity equation (2) within the time integration routine.

Step 1 is isolated from 2 and 3 so that the vertical transport calculation (step 2) and its application (step 3) is independent of the "prescription" for determining the target layer thickness (step 1). Thus, new algorithms for determining layer position can be readily implemented and tested. In addition, this partitioning insures that $w_{k}^{t}$ is used consistently in the time-stepping algorithm for both volume and tracer evolution (3). This is the Lagrangian treatment of the vertical direction described in Adcroft and Hallberg (2006), where layer thickness is prognostic and sea surface height is diagnostic. For fully Lagrangian layers, the vertical transport is set to zero in step 2. This setting is used for isopycnal simulations.

The desired ALE thickness in step 1 includes contributions from four terms (4):

1. Resting thickness, $h^{\text {rest }}$, is the layer thickness when the ocean is at rest, i.e. without SSH or internal perturbations. For z-type coordinates, the resting thickness is constant in each horizontal layer, and cells below bathymetry are unused. For sigma coordinates, all layers are used, and $h^{\text {rest }}$ varies horizontally in proportion to the column's total depth.

2. SSH alteration, $h^{S S H}$, alters layer thicknesses so that changes are in proportion to the sea surface height $(\mathrm{SSH})$,

$$
h_{k}^{S S H}=\zeta \frac{W_{k} h_{k}^{r e s t}}{\sum_{k^{\prime}=1}^{k m a x} W_{k^{\prime}} h_{k^{\prime}}^{r e s t}} .
$$


The weights $W_{k}$ determine how SSH oscillations are distributed amongst the layers, as described in Table 3.

3. High-frequency thickness, $h^{h f}$, allows high-frequency thickness oscillations, such as internal gravity waves, to be treated in a Lagrangian manner. This is the "z-tilde" scheme of Leclair and Madec (2011) described in the next section.

4. Minimum/maximum thickness alteration, $h^{\text {limit }}$, is the change in thickness required to enforce the minimum and maximum thickness in each cell. For example, when a cell is too thin, $h^{\text {limit }}$ is positive, while nearby cells in the vertical incur a corresponding negative $h^{\text {limit }}$ to conserve volume in the column.

Of the four terms, resting thickness is always positive, while the others are small alterations about zero. Summing (4) over a column,

$$
\begin{aligned}
\sum_{k=1}^{k \max } h_{k}^{A L E} & =\sum_{k=1}^{k \max } h_{k}^{\text {rest }}+\sum_{k=1}^{k \max } h_{k}^{S S H}+\sum_{k=1}^{k \max } h_{k}^{h f}+\sum_{k=1}^{k \max } h_{k}^{\text {limit }} \\
& =H+\zeta+0+0 .
\end{aligned}
$$

Thus the first two terms are always included so that the column thickness sums to $H+\zeta$, while the second two terms are optional.

The MPAS-Ocean ALE algorithm broadens the choices in vertical coordinate settings from traditional vertical coordinate names. For example, the choice of weights in (6) may place all SSH oscillations in the top layer only ( $W_{1}=1, W_{k}=0$ otherwise), or distribute $\mathrm{SSH}$ oscillations amongst all layers, in proportion to the resting thickness $\left(W_{k}=1\right.$ for all $\left.k\right)$. If the initial layer thicknesses are constant in the horizontal, these settings correspond to z-level and z-star vertical coordinates. A third option is weighted z-star, with user-specified weights for $W_{k}$ (Table 3), where one could specify $W_{k}=1$ in the upper layers and taper $W_{k}$ off to zero at depth, so that SSH oscillations primarily affect the mixed layer. The advantage of z-star and weighted z-star is that layers may be much thinner than the maximum $\mathrm{SSH}$, which is typically $1-2 \mathrm{~m}$. The high-frequency thickness (z-tilde) and minimum/maximum thickness alteration may be applied in addition to the other settings. Simulations presented as z-tilde in this paper use SSH alteration weights of $W_{k}=1$ for all layers, so it is effectively z-tilde applied to a z-star vertical coordinate. Partial bottom cells (PBCs) may also be used independent of the other vertical coordinate settings. The overflow test case was conducted with and without PBCs, and all global simulations use PBCs. 
Table 3: Vertical coordinate settings for traditional names. The flag column is the MPASOcean setting for the configuration flag config_vert_coord_movement

\begin{tabular}{ccc}
\hline \hline name & weights & flag \\
\hline z-level & $W_{k}=\left\{\begin{array}{cc}1 k=1 \\
0 k>1\end{array}\right.$ \\
Z-star & $W_{k}=1 \forall k$ \\
weighted z-star & any $W_{k}$ & 'fixed' \\
\hline
\end{tabular}

Table 4: Variables used in ALE equation sets. A subscript $k$ indicates the layer index. The $\nabla$ indicates a horizontal gradient within a single layer.

\begin{tabular}{ccc}
\hline \hline symbol & name & notes \\
\hline$D$ & thickness-weighted divergence & see $(8)$ \\
$\bar{D}$ & barotropic divergence & see $(9)$ \\
$D^{\prime}$ & baroclinic divergence & $D_{k}^{\prime}=D_{k}-\bar{D}$ \\
$D^{l f}$ & low-frequency divergence & see $(12)$ \\
$D^{h f}$ & high-frequency divergence & $D_{k}^{h f}=D_{k}^{\prime}-D_{k}^{l f}$ \\
$h^{A L E}$ & desired ALE thickness & see $(4)$ \\
$h^{r e s t}$ & resting thickness & see $(6)$ \\
$h^{S S H}$ & SSH thickness alteration & see $(13)$ \\
$h^{h f}$ & high-freq. thickness alteration & \\
$h^{\text {limit }}$ & min/max thickness alteration & $k \sum_{k=1}^{k m a x} h_{k}^{r e s t}$ \\
$H$ & total resting thickness & $H$ \\
$k m a x$ & index of deepest water cell & \\
$W$ & SSH thickness weights & constant \\
$\tau_{D l f}$ & frequency filter time scale & constant \\
$\tau_{h h f}$ & restoring time scale for $h^{h f}$ & constant \\
$\kappa_{h h f}$ & $h^{h f}$ diffusion & $\sum_{k=1}^{\sum} h_{k}-H$ \\
$\zeta$ & sea surface height & $\zeta$
\end{tabular}

\subsection{High-frequency thickness algorithm (z-tilde)}

The high-frequency thickness alteration, $h^{h f}$, in (4) allows layer thickness to oscillate so that high-frequency motions, such as internal gravity waves, are treated in a Lagrangian manner. Low-frequency motions, such as seasonal 
changes or slow motions of water masses, are treated in an Eulerian manner. This is the "z-tilde" scheme of Leclair and Madec (2011), which generally reduces spurious vertical mixing and better preserves water mass properties.

To begin, consider the horizontal divergence in each cell,

$$
D_{k}=\nabla \cdot\left(h_{k} \mathbf{u}_{k}\right) \text {. }
$$

In each column the barotropic divergence is the vertical sum,

$$
\bar{D}=\frac{\sum_{k=1}^{k \max } h_{k} D_{k}}{\sum_{k=1}^{k \max } h_{k}},
$$

so that the divergence in a cell may be written as the sum of the barotropic part and the remaining baroclinic part,

$$
D_{k}=\bar{D}+D_{k}^{\prime} .
$$

Furthermore, the baroclinic divergence is split between a low-frequency barclinic divergence and a high-frequency baroclinic divergence,

$$
D_{k}^{\prime}=D_{k}^{l f}+D_{k}^{h f} .
$$

Two additional prognostic equations are solved in this configuration,

$$
\begin{gathered}
\frac{\partial D_{k}^{l f}}{\partial t}=-\frac{2 \pi}{\tau_{D l f}}\left(D_{k}^{l f}-D_{k}^{\prime}\right) \\
\frac{\partial h_{k}^{h f}}{\partial t}=-D_{k}^{h f}-\frac{2 \pi}{\tau_{h h f}} h_{k}^{h f}+\nabla_{h} \cdot\left(\kappa_{h h f} \nabla_{h} h_{k}^{h f}\right),
\end{gathered}
$$

where the first is a simple low-pass filter for the baroclinic divergence and the second is a prognostic equation for the high-frequency thickness, and variables are defined in Table 4 . The filter time scale $\tau_{D l f}$ is typically set to five days, so that internal gravity waves and other short time scale adiabatic phenomena are included in the high-frequency divergence, $D^{h f}$. This is computed from the low-frequency divergence $D^{l f}$ with the simple diagnostic equations (8-11). The high-frequency thickness equation is forced by the high-frequency divergence, and includes a restoring term and horizontal diffusion term to avoid long-term drift of $h^{h f}$ from zero. The restoring time 
scale, $\tau_{h h f}$, is typically set to 30 days, and the horizontal diffusion is zero for the simulations in this paper. However, these two terms are ad-hoc methods to ensure reasonable vertical grids, and may need to be adjusted for other domains.

The enforcement of a minimum and maximum layer thickness in (4) is an addition to the algorithm presented in Leclair and Madec (2011) that was needed to run test cases with large initial perturbations. In those situations, a high frequency divergence would include the evacuation of all mass from some cells. This can be avoided with the simple minimum thickness requirement. In realistic global simulations starting from climatological initial conditions, the minimum and maximum were often not required, but are still a useful safeguard against very thin layers when the dynamics occur faster than the restoring time scale. An optional maximum layer thickness is included to prevent severely distorted layers, and was required to run z-tilde in domains with strongly transient flows.

\subsection{Resting Potential Energy}

The primary metric used in this study to diagnose spurious mixing is the resting potential energy (RPE), where the potential density $\rho(x, y, z)$ is sorted and redistributed in the model domain (Ilicak et al., 2012). The sorted density, $\rho^{*}(z)$, is constant horizontally and monotonically increasing with depth. The RPE is

$$
R P E=g \int_{\Omega} z \rho^{*}(z) d V,
$$

where $\Omega$ is the domain. An equally valid interpretation of RPE is a measure of the fluid's vertical center of mass. Vertical mixing results in a monotonicallyincreasing center of mass with time. Thus, when surface buoyancy forcing is absent, as is the case in the simulations presented below, the time-rate-of change of RPE is a measure of vertical mixing. A visual example is that RPE increases as more cells are in the mid-range of the color bars in Figures 1 and 4.

An important limitation of this method is that it is only valid for cases with zero buoyancy fluxes. Thus all cases presented here are spin-down simulations from an initial condition or, for real-world cases, from an equilibriated condition. The RPE method cannot be used to diagnose mixing in typical realistic simulations that include surface forcing. 
The algorithm for computing RPE for discrete fields is as follows. For each cell in the domain, there is an associated potential density $\rho(i, k)$ and volume $V(i, k)$ where $i$ and $k$ are horizontal and vertical indices. After sorting, the new density $\rho^{*}(j)$ is monotonically increasing, and the volume $V^{*}(j)$ must be rearranged in the same way, so that each $\rho^{*}$ corresponds to the same cell volume as in the original variables (note $V^{*}$ is not sorted to be monotonically increasing). Each cell is now given a new mid-depth $z^{*}(j)$, horizontal area $A^{*}(j)$, and thickness $h^{*}(j)=A^{*}(j) / V^{*}(j)$ by piling thin cells in a stack from the top to the bottom of the domain. The horizontal area is the crosssectional area of the domain at a depth of $z^{*}$. The RPE is then computed as

$$
R P E=g \sum_{j} z^{*}(j) \rho^{*}(j) V^{*}(j),
$$

which has units of Joules. The initial value RPE of a particular simulation depends on the domain and initial conditions, and so is not relevant to the mixing. Thus plots of RPE use values normalized to time zero,

$$
\frac{R P E(t)-R P E(0)}{R P E(0)} .
$$

This same argument holds for the time derivative of RPE, but the nonnormalized value is used to compare to data published in Ilicak et al. (2012). Plots showing $d R P E / d t$ use units of $\mathrm{W} \mathrm{m}^{-2}$, and are normalized only by the surface area of the domain.

Real-world cases use the nonlinear equation of state by Jackett and McDougall (1995), where density is a function pressure as well as temperature and salinity. Thus potential density was used for $\rho^{*}$ in (15), following the $\rho_{2000}$ method in Ilicak et al. (2012) [Appendix A]. (We used potential density referenced to the surface, but this is a minor difference.) This method of RPE calculation does not account for changes in potential density due to pressure after the potential density is resorted to a new depth. That would require an expensive iterative algorithm, which has been shown to change RPE by less than 1\% (Ilicak et al., 2012, Appendix A). See Saenz et al. (2014) for an alternative efficient method that accounts for pressure changes when resorting potential density. An additional issue with real-world cases is that our RPE computation allows water parcels to be sorted throughout the world's oceans without constraints due to the bathymetry of ocean basins. Sorting density separately within distinct basins would change the value of 
RPE, but would not alter relative mixing rates when comparing simulations using the same domain.

For real-world simulations the mean density and depth must be subtracted before computing the RPE (Ilicak et al., 2012, Appendix A). Define $V=\int_{\Omega} d V, \bar{\rho}=\frac{1}{V} \int_{\Omega} \rho d V$, and $\bar{z}=\frac{1}{V} \int_{\Omega} z d V$, so that the perturbation variables are $\rho^{\prime}=\rho-\bar{\rho}$ and $z^{\prime}=z-\bar{z}$. Then (15) is revised to be

$$
R P E=g \sum_{j}\left(z^{*}\right)^{\prime}(j)\left(\rho^{*}\right)^{\prime}(j) V^{*}(j) .
$$

Note that the order of operations of sorting and subtracting the mean are interchangeable.

Idealized test cases use a harmonic operator for horizontal momentum diffusion of the form $\nu_{h} \nabla^{2}$. In this case, RPE results are shown as a function of the grid Reynolds number,

$$
R e_{\Delta}=\frac{U \Delta x}{\nu_{h}},
$$

where $U$ is the characteristic velocity scale, $\Delta x$ is the horizontal mesh spacing, and $\nu_{h}$ is the specified horizontal viscosity with units of $\mathrm{m}^{2} / \mathrm{s}$ (Bryan et al., 1975; Griffies, 2004; Ilicak et al., 2012). For the lock exchange and overflow test cases, the velocity scale is the theoretical wave speed in a rectangular channel; for the internal wave and baroclinic eddies test cases, it is computed from the root-mean-squared velocity computed over the domain.

For real-world simulations, a horizonal biharmonic operator is used, with coefficient $\nu_{4 h}$ and units $\mathrm{m}^{4} / \mathrm{s}$. Then the grid Reynolds number is

$$
R e_{\Delta}=\frac{U(\Delta x)^{3}}{8 \nu_{4 h}}
$$

where the velocity scale $U$ is computed from the globally averaged kinetic energy (Griffies and Hallberg, 2000).

\section{Idealized test cases}

In this section we evaluate vertical mixing in MPAS-Ocean using four idealized test cases introduced in Ilicak et al. (2012), and make direct comparisons to other ocean models under identical conditions whenever the data is available. Parameters that vary amongst the four idealized test cases are 
listed in Table 5. The remainder of this section describes settings that are the same in all four idealized test cases.

The tracer advection scheme, which is evaluated in detail in Section 3.6, is monotonic flux-corrected transport (Skamarock and Gassmann, 2011), with third-order reconstruction of the tracer values at the cell edges. The timestepping algorithm is split-explicit, with the long baroclinic time step $(\Delta t)$ given in Table 5. The two-dimensional barotropic stage uses 40 subcycles to step through $2 \Delta t$, as described in Appendix A5 of Ringler et al. (2013). The horizontal turbulence closure for these cases is

$$
\mathbf{D}_{h}^{u}=\nu_{h} \nabla^{2} \mathbf{u}
$$

for momentum, with a constant viscosity $\nu_{h}$, and $\mathbf{D}_{h}^{\phi}=0$ for tracers. There is no surface forcing by winds, tracer fluxes, or tracer restoring. Further details of how these settings are applied in MPAS-Ocean can be found in Jacobsen et al. (2013).

The idealized domains are in $(x, y, z)$ Cartesian space, and individual cells are regularly spaced hexagons. A handful of tests were conducted using quadrilateral cells as well, with nearly identical results. The lock exchange, overflow, and internal wave test cases (1-3) are effectively two-dimensional in $(x, z)$, but simulations are three-dimensional and use four cells with periodic boundaries in the y-direction. For this study, a single resolution was sufficient for each of cases 1-3, and three horizontal resolutions were used for the baroclinic eddies, case 4 (see grid cell size in Table 5). This is a reduction from the number of horizontal and vertical resolutions presented in Ilicak et al. (2012). The domains are partitioned into blocks to run on multiple processors, ranging in number from eight for the lock exchange to several hundred for the $1 \mathrm{~km}$ baroclinic eddies case.

All four idealized test cases use a linear equation of state of the form

$$
\rho(\Theta, S)=\rho_{r e f}-\alpha\left(\Theta-\Theta_{r e f}\right)+\beta\left(S-S_{r e f}\right)
$$

where $\rho_{\text {ref }}=1000 \mathrm{~kg} \mathrm{~m}^{-3}, \alpha=0.2 \mathrm{~kg} \mathrm{~m}^{-3} \mathrm{C}^{-1}$, and $\Theta_{\text {ref }}=5 \mathrm{C}$. The initial salinity is set uniformly as $S=S_{r e f}$ so that density depends only on temperature. For a linear equation of state there is no explicit depth or pressure dependence, so potential density is identical to density. All cases begin with zero velocity. 
Table 5: Parameter settings for idealized test cases. Isopycnal and z-type initial conditions are specified by $\rho$-layer and z-layer, respectively. RPE avg is the time over which $d R P E / d t$ was averaged.

\begin{tabular}{|c|c|c|c|c|}
\hline & $\begin{array}{c}\text { case } 1 \\
\text { lock exchange }\end{array}$ & $\begin{array}{c}\text { case } 2 \\
\text { overflow }\end{array}$ & $\begin{array}{c}\text { case } 3 \\
\text { internal wave }\end{array}$ & $\begin{array}{c}\text { case } 4 \\
\text { baroclinic eddies }\end{array}$ \\
\hline domain size $x$ & $64 \mathrm{~km}$ & $200 \mathrm{~km}$ & $250 \mathrm{~km}$ & $160 \mathrm{~km}$ \\
\hline domain size $y$ & - & - & - & $500 \mathrm{~km}$ \\
\hline domain size $z$ & $20 \mathrm{~m}$ & $2000 \mathrm{~m}$ & $500 \mathrm{~m}$ & $1000 \mathrm{~m}$ \\
\hline grid cell size & $0.5 \mathrm{~km}$ & $1 \mathrm{~km}$ & $5 \mathrm{~km}$ & $1,4,10 \mathrm{~km}$ \\
\hline number $\rho$-layers & 2 & 2 & 20 & - \\
\hline number z-layers & 20 & 100 & 20 & 20 \\
\hline z-layer thickness & $1 \mathrm{~m}$ & $20 \mathrm{~m}$ & $25 \mathrm{~m}$ & $50 \mathrm{~m}$ \\
\hline time step $\Delta t, \mathrm{~s}$ & $30-120$ & $5-20$ & 300 & $30,120,300$ \\
\hline$\nu_{h}, \mathrm{~m}^{2} / \mathrm{s}$ & $0.01,0.1,1$ & $0.01,0.1,1$ & $0.01,1$ & $1,5,10$ \\
\hline & $10,100,200$ & $10,100,1000$ & 15,150 & 20,200 \\
\hline$\nu_{v}, \mathrm{~m}^{2} / \mathrm{s}$ & 0.0001 & 0.0001 & 0.0001 & 0.0001 \\
\hline$\kappa_{h}, \mathrm{~m}^{2} / \mathrm{s}$ & 0 & 0 & 0 & 0 \\
\hline$\kappa_{v}, \mathrm{~m}^{2} / \mathrm{s}$ & 0 & 0 & 0 & 0 \\
\hline bottom drag & - & - & - & 0.01 \\
\hline Coriolis, $\mathrm{s}^{-1}$ & - & - & - & $-1.2 \times 10^{-4}$ \\
\hline run duration & $17 \mathrm{hrs}$ & $40 \mathrm{hrs}$ & 200 days & 320 days \\
\hline RPE avg & $17 \mathrm{hrs}$ & $13-37$ hrs & 10-100 days & 1-320 days \\
\hline$R e_{\Delta}$ velocity & $u_{f}$ & $u_{f}$ & $U_{c}$ & $U_{c}$ \\
\hline
\end{tabular}

\subsection{Lock exchange test case}

The lock exchange case provides a way to measure mixing in the simplest possible configuration. The initial condition,

$$
\Theta(x, z)=\left\{\begin{array}{cl}
5 & x<32 \mathrm{~km} \\
30 & x \geq 32 \mathrm{~km}
\end{array}\right.
$$

may be thought of as two basins of water that are allowed to interact at time zero. Warm water flows over the cold water from right to left, and vice versa. Simulations were performed for a range of horizontal viscosities, while the vertical viscosity is kept constant and explicit tracer diffusion is zero (Table 5). Higher viscosities generate a smooth, tilted interface between the warm and cold fluid, and produce less mixing, as quantified by a lower 
time-rate-of-change in RPE. Simulations with lower viscosities are more energetic, have a higher grid Reynolds number, and produce more oscillations in the temperature field (Figures 1 and 2). The stability condition to prevent spurious numerical modes and unphysical extrema is $R e_{\Delta}<2$ (Griffies, 2004). Comparing grid Reynolds numbers of 2.5 and 250 in Figure 1 shows that the later contains grid-scale noise, and is not well-resolved. The time step of the high viscosity runs were limited by the viscous CFL condition; a time step of 120 seconds was used for lower viscosities, but was decreased to 60 and 30 seconds at $\nu_{h}=100$ and $200 \mathrm{~m}^{2} \mathrm{~s}^{-1}$, respectively. All models considered here are hydrostatic, so the vertical momentum equation lacks an acceleration term. Non-hydrostatic simulations would include the generation of Kelvin-Helmholtz billows (Fringer et al., 2006).

Because all diffusion has been turned off, the correct solution is where no mixing occurs, and the front propagates with no intermediate temperatures between $5 \mathrm{C}$ and $30 \mathrm{C}$. This is demonstrated by MPAS-Ocean in isopycnal mode and the GOLD simulations in Ilicak et al. (2012), where the RPE remains constant. The MPAS-Ocean isopycnal simulations used only two layers, with temperatures of 5C and 30C. MPAS-Ocean does not support massless layers, so the initial thickness is $1 \mathrm{~cm}$ for the warmer top layer in the left half of the domain, and $19.99 \mathrm{~m}$ in the right half, while the cold bottom layer is opposite. As the isopycnal simulations proceed, the thin layers fill at the wave front (Figure 1). There is zero vertical advection between the two layers, so the temperature of each layer remains constant in time. The shape of the layer interface varies with grid Reynold's number, where the interface changes from tilted to flat as the grid Reynold's number increases.

The amount of mixing by MPAS-Ocean in z-star mode is less than MIT$\mathrm{gcm}$ and MOM, but more than ROMS (Figure 2a). The ROMS low value of $R P E$ is likely due to its third-order, upstream-biased, dissipative advection scheme for momentum (Shchepetkin and McWilliams, 2005), which adds local dissipation to enforce stability. The time derivative of RPE at 17 hours is lower for MPAS-Ocean than for MITgcm over the range of viscosities tested (Figure 2c). Results for z-level and z-star vertical coordinate settings in MPAS-Ocean are nearly identical, as one would expect for an internally propagating wave.

Using Bernoulli's equation for an ideal fluid, the speed of a gravity current in a rectangular channel is

$$
u_{f}=\frac{1}{2} \sqrt{g H\left(\delta \rho / \rho_{0}\right)}
$$


(Benjamin, 1968), which is $0.496 \mathrm{~m} / \mathrm{s}$ for this test problem. This predicts that the right front location is at $62.4 \mathrm{~m}$ at 17 hours. The front in MPASOcean simulations is at $60 \mathrm{~m}$ and $62 \mathrm{~m}$ for the $\nu_{h}=1 \mathrm{~m}^{2} / \mathrm{s}$ and $\nu_{h}=100$ $\mathrm{m}^{2} / \mathrm{s}$, respectively (Figure 1 ), which is very close to the front location in MITgcm and MOM simulations (Ilicak et al., 2012, Figure 5). The wave front propagates slightly faster in isopycnal models. The theoretical wave speed $u_{f}$ is used to compute the grid Reynolds number in (18). The root-meansquared velocity, computed over the domain, begins at zero and increases to $0.5 \mathrm{~m} / \mathrm{s}$ at 16 hours, so is in good agreement with $u_{f}$.

Overall, the lock exchange allows modelers to test mixing and advection without the complications of topography, Coriolis forces, bottom drag, and any surface forcing. The remaining test cases add these in one by one, so that the model developer may gain confidence through step-wise validation exercises. Mixing rates are highly sensitive to the choice of advection scheme, as will be shown in Section 3.6.

\subsection{Appropropriate use of z-tilde}

The z-tilde scheme was tested in the lock exchange with both the zlevel and isopycnal initial conditions described in the previous section. The results depend on the frequency filter time scale, $\tau_{D l f}$ (Figure 3 ). The results for z-level initial conditions (20 one-meter-thick layers) are as follows. When $\tau_{D l f}<1$ hour, the wave dynamic, which is moving at the time scale of several hours, is filtered out of the high-frequency thickness, and the grid remains at its initial Eulerian position. In this case, $d R P E / d t$ values converge to the z-level values as $\tau_{D l f}$ goes to zero. At the other extreme, when $\tau_{D l f}>$ 12 hours, the wave is included in the high frequency thickness, and the grid behaves in a Lagrangian manner: as the wave proceeds from the center of the domain, layers behind the wave fill to the maximum layer thickness, and those in the remainder of the column evacuate to the minimum layer thickness (typically set to $10 \mathrm{~m}$ and $0.1 \mathrm{~m}$, respectively). In this case, the grid becomes very distorted with oscillations, and produces higher $d R P E / d t$ values than the original z-level simulations. When $\tau_{D l f}$ is between 1 and 12 hours, the grid moves in a weakly Lagrangian manner, and never reaches the minimum or maximum thickness. In this case, the grid is less distorted, and there is some reduction in mixing.

When the initial conditions are isopycnal (i.e. two layers, each with constant temperature) $d R P E / d t$ values can be made arbitrarily small by increasing $\tau_{D l f}$, until it converges to a purely Lagrangian grid where $d R P E / d t=0$. 

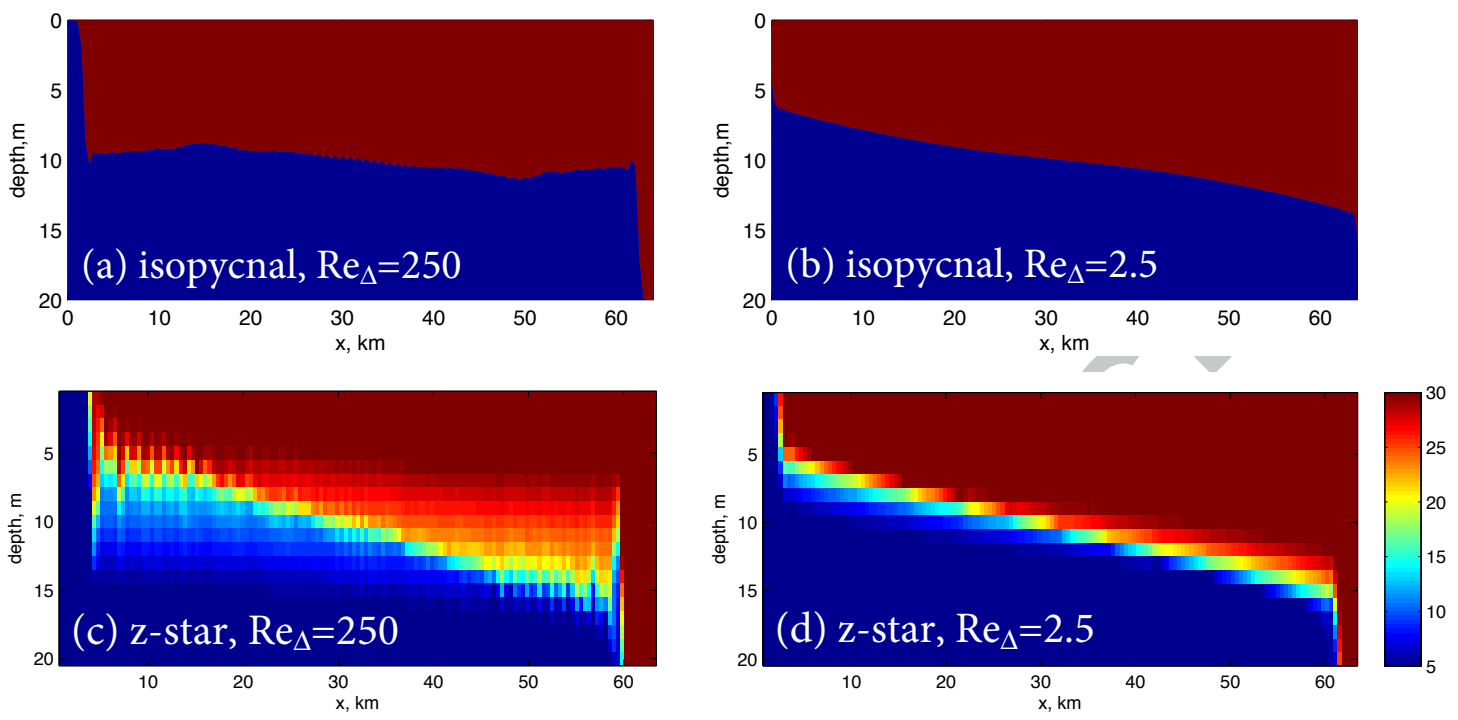

Figure 1: Lock exchange test case: Temperature, degrees C, at 17 hours for MPAS-Ocean using isopycnal (a, b) and z-star vertical coordinates $(\mathrm{c}, \mathrm{d})$. The horizontal viscosity is $\nu_{h}=1.0(\mathrm{a}, \mathrm{c})$ and $\nu_{h}=100(\mathrm{~b}, \mathrm{~d})$, corresponding to grid Reynolds numbers of $\operatorname{Re}_{\Delta}=250$ and $R e_{\Delta}=2.5$. In (b), the wave front has just reached the edge of the domain at 17 hours.

When $\tau_{D l f}<100$ days, the grid moves move slowly, and the thick (originally isopycnal) layers on the left and right experience a great deal of mixing.

The application of z-tilde to the lock exchange shows that z-tilde is not appropriate for test cases with strongly transient flows. When the grid follows fast dynamics in a Lagrangian manner, those dynamics must be small perturbations or the grid will be strongly distorted, resulting in similar or higher mixing rates compared to an Eulerian grid. Adding high-frequency thickness diffusion $\left(\kappa_{h h f}\right)$ improves the smoothness of the grid, but does not substantially reduce the mixing. The only way to have the z-tilde scheme converge to zero mixing in the Lagrangian limit of high $\tau_{D l f}$ is to use isopycnal-layer initial conditions. Then z-tilde reduces to an isopycnal-layer model, but this defeats the purpose of z-tilde, which is meant to reduce mixing for small, fast perturbations within z-level-type vertical coordinates.

\subsection{Overflow test case}

The overflow test case is similar to the lock exchange, but includes a drop in topography that induces vertical advection and mixing. The bottom 
depth, shown in Figure 4, is

$$
d(x)=d_{1}+\frac{1}{2}\left(d_{2}-d_{1}\right)\left(1+\tanh \left(\frac{x-x_{0}}{\sigma}\right)\right)
$$

with $d_{1}=500 \mathrm{~m}, d_{2}=2000 \mathrm{~m}, x_{0}=40 \mathrm{~km}$, and $\sigma=7 \mathrm{~km}$. The initial temperature is

$$
\Theta(x, z)= \begin{cases}10 & x<20 \mathrm{~km} \\ 20 & x \geq 20 \mathrm{~km}\end{cases}
$$

As the wave descends the steep topography, mixing depends on the horizontal viscosity, the advection scheme, the vertical coordinate, and the treatment of the bottom topography. Like the lock exchange, low horizontal viscosity results in higher kinetic energy and higher Reynolds number. At low viscosity the interface between warm and cold water has more oscillations (Figure 4). The higher kinetic energy induces more mixing, resulting in higher RPE at higher Reynolds numbers (Figure 5). Z-level and z-star vertical coordinates produce nearly identical results. The overflow was also tested in isopycnal and sigma coordinates, but not z-tilde, for the same reasons as the lock exchange (Section 3.2).

Simulations with MPAS-Ocean isopycnal coordinates use two layers, each with a uniform temperature of $10 \mathrm{C}$ and $20 \mathrm{C}$. The inactive layer is initialized with a layer thickness of $1 \mathrm{~cm}$ for high viscosities, and $1 \mathrm{~m}$ for low viscosities. These layers are allowed to activate when the average thickness of a cell and its neighbor is greater than $2 \mathrm{~cm}$ and $2 \mathrm{~m}$, respectively. This check prevents the inactive layer from descending the slope prematurely. In addition, a minimum layer thickness of $1 \mathrm{~cm}$ to $1 \mathrm{~m}$ is enforced once the layer is activated. Smaller timesteps, in some cases down to one second, were required to prevent negative layer thickness just behind the head. A bottom drag coefficient of 0.01 was required for the isopycnal simulations to run stably.

In order to show the versatility of the MPAS-Ocean ALE vertical coordinate, we include terrain-following sigma-coordinate simulations of the overflow test case (Figure 4i-l). The other test cases are flat-bottom, so sigma and z-star would be identical. When the overflow test case is configured with a sigma coordinate, the 100 layers are uniformly distributed between the ocean surface and ocean bottom. This produces layer thicknesses that are $5 \mathrm{~m}$ over the shelf and $20 \mathrm{~m}$ in the full-depth region. These sigma coordinate tests include the pressure gradient correction due to tilted coordinate surfacesthis is the $\nabla z_{k}^{\text {mid }}$ term in (1), computed as in Shchepetkin and McWilliams 
(2003, Section 2, method 4). Weighted averaging of the density and vertical coordinate (Song, 1998; Shchepetkin and McWilliams, 2003, Section 3) is not included. Errors in sigma coordinate models due to the compressibility of sea water (Shchepetkin and McWilliams, 2003, Section 7) are not relevant for this test case, which uses a linear equation of state.

The flow begins as a wave front in a rectangular channel, with a theoretical velocity of $u_{f}=1.57 \mathrm{~m} / \mathrm{s}$ from (23) using $H=500 \mathrm{~m}$ for the upper shelf. This places the wave front at $37 \mathrm{~km}$ at 3 hours, which is near the simulated wave front in all the models, as shown in Figure 4 and Ilicak et al. (2012, Figure 9). By six hours, the wave front has reached the ridge bottom, but locations vary among models: $47 \mathrm{~km}$ for MPAS-Ocean, $55 \mathrm{~km}$ for MOM, 60 $\mathrm{km}$ for GOLD, $70 \mathrm{~km}$ for MITgcm, and $78 \mathrm{~km}$ for MPAS-Ocean with sigma coordiantes (all using $\nu_{h}=1000 \mathrm{~m}^{2} / \mathrm{s}$ ). Unlike the lock exchange test case, there is no theoretical solution to compare to for the head location of the overflow, so it is difficult to say which models are more accurate. At high viscosity, the overflow plug of water keeps a coherent shape as it descends, with significant mixing at the head. At low viscosity, the cold water is no longer a continuous plug, but is completely dispersed with waves behind the head for many of the models. Z-level and z-star vertical coordinates induce more mixing, which slows the descent down the slope. MPAS-Ocean isopycnal and sigma coordinate simulations descend further in the same amount of time.

All overflow simulations were conducted with zero horizontal and vertical tracer diffusion and a small value of vertical viscosity $\left(\nu_{v}=10^{-4}\right)$. In MPASOcean z-level and z-star coordinates, the cold wave head remained above several cells of warm water as it initially descended down the slope (Figure $4 \mathrm{a}, \mathrm{c})$. This behavior is more apparent in MPAS-Ocean at three hours than in MITgcm and MOM, and may be responsible for the head location of MPASOcean being further left at nine hours than the other models. Typically ocean models use a convective adjustment scheme or a Richardson number-based vertical mixing to avoid unstable density profiles, but these are not used here in order to measure only spurious mixing. Some simulations using z-star and partial bottom cells did not run to completion due to this instability (Figure $\left.5, R e_{\Delta}>100\right)$, but proceed normally when Richardson number-based vertical mixing is added (not shown).

Mixing rates, as measured by $d R P E / d t$, are highest for z-star using full cells, lower for z-star with partial bottom cells, and lowest for sigma coordinate simulations (Figure 5). This progression is expected, because full cells 
have the largest vertical steps to cause mixing. In sigma coordinates, the cold head falls down the slope by advecting horizontally in grid space, so much less mixing is induced. One exception is the higher value at $R e_{\Delta}=1$, which appears to be caused by interaction with the right wall. MITgcm and MOM mixing rates lie between the MPAS-Ocean z-star and sigma values. In all the high viscosity cases, the head hits the right wall at $200 \mathrm{~km}$ before 37 hours, so some mixing in Figure 5 is due to wall interactions. In low viscosity cases, the head is sufficiently dispersed that it never reaches the right wall. The time window of 13-37 hours was used in order to make comparisons with the other ocean models.

\subsection{Internal waves test case}

Internal waves are ubiquitous in the ocean. They are driven by winds, tides, and surface pressure oscillations, and propagate through the stratified ocean. Breaking internal gravity waves are a significant source of mixing in ocean observations (Alford, 2003), but linear internal waves tend to produce vertical mixing in ocean models, particularly in those with fixed vertical levels such as z-level and z-star (Gouillon, 2010). Models that can follow isopycnal surfaces in a Lagrangian manner, such as those with isopycnal and hybrid-isopycnal coordinates, can significantly reduce excessive mixing because layers move with the internal waves and restrict fluid transport between layers. The z-tilde scheme restricts mixing in a similar way, but may be applied to level-type coordinates.

The lock exchange and overflow test cases begin with only two values of temperature, and so are extreme circumstances to measure a model's behavior. The internal wave test case is vertically stratified, begins with a small perturbation, and induces internal wave propagation similar to that found in realistic global simulations. This test has the advantage that the simulation may proceed for hundreds of days, rather than 20-40 hours as is the case for the lock exchange and overflow, so that mixing may be measured over a long period.

The initial temperature distribution is $\Theta_{0}(z)+\Theta^{\prime}(x, z)$, which has a background stratification of

$$
\Theta_{0}(z)=\Theta_{b o t}+\left(\Theta_{t o p}-\Theta_{b o t}\right) \frac{z_{b o t}-z}{z_{b o t}}
$$

where $\Theta_{b o t}=10.1 \mathrm{C}, \Theta_{\text {top }}=20.1 \mathrm{C}$, and $z_{\text {bot }}=-487.5 \mathrm{~m}$ (note sign conven- 
tion on $z$ ). The initial wave is

$$
\Theta^{\prime}(x, z)=-A \cos \left(\frac{\pi}{2 L}\left(x-x_{0}\right)\right) \sin \left(\pi \frac{z+\Delta z / 2}{z_{b o t}+\Delta z / 2}\right)
$$

for $x_{0}-L<x<x_{0}+L$, where $L=50 \mathrm{~km}, x_{0}=125 \mathrm{~km}$, and $\Delta z=25$ $\mathrm{m}$. We present a single case with $A=2.0 \mathrm{C}$, which is the high amplitude case shown in Ilicak et al. (2012, Figure 11b). The perturbation is a single hump of displaced isotherms that initiate symmetric waves propagating out from the center. The waves meet the solid (non-periodic) boundary after a day, and then return to the center, forming higher wavenumber modes as the waves interact and numerical dispersion takes place. After 200 days the domain is still active with internal waves, but the models differ substantially in their shapes and positions, as shown in Figure 6, and Ilicak et al. (2012, Figure 11).

As in the first two test cases, mixing is a strong function of horizontal viscosity (Figure 7b); RPE increases in time and with lower viscosity. This is summarized in Figure 7c, where each simulation is represented by a symbol. The grid Reynolds number is computed using (18) with a characteristic velocity of $U=\sqrt{ } 2 K E$, where $K E$ is the average kinetic energy from 10-100 days. Isopycnal models such as GOLD have zero mixing for the duration of the simulation. MPAS-Ocean, MITgcm, and MOM have similar mixing for the first 50 days, and diverge somewhat thereafter (Figure 7a). MPAS-Ocean results are identical for z-level and z-star vertical coordinate settings because internal waves do not influence the sea surface height. This case was also tested in isopycnal coordinates, using 20 layers of constant temperature.

The internal wave case is the perfect test for the z-tilde scheme, introduced in Section 2.2. In the z-tilde scheme, layers move vertically with the internal waves, as they do in an isopycnal model. Layers are initially level and equally spaced for all simulations. Animations of z-tilde simulations show the grid moving with the internal waves, so that by 200 days the grid nearly follows the temperature profile (Figure 6). In z-tilde mode, the grid may be initialized as level layers, or the initial layers may follow density contours.

The main parameter in the z-tilde scheme is the frequency filter time scale $\tau_{D l f}$. When it is smaller than the physical time scales in the model, everything is included in the low-frequency component, so the high-frequency thickness alteration $\left(h^{h f}\right)$ approaches zero. In this case the grid does not move and the model behaves as it would with z-star. As the filter time scale is increased, 
more of the physical processes are included in the high frequency divergence, the grid moves more, and mixing is reduced. The internal wave test provides an illustrative example of how spurious mixing may be reduced by increasing the filter time scale (Figure 7). All z-tilde tests used a time step of $300 \mathrm{~s}$, a high-frequency thickness restoring timescale $\left(\tau_{h h f}\right)$ of 30 days, and no high frequency thickness diffusion $\left(\kappa_{h h f}=0\right)$.

The vertical cross-coordinate transport is correlated with RPE, and provides an indirect measure of mixing; in a stratified fluid, vertical crosscoordinate transport induces mixing. Vertical cross-coordinate transport is reduced as the filter time scale increases (Figure 8). Note that this measure is different from vertical velocity; in a fully Lagrangian vertical coordinate, vertical cross-coordinate transport is zero, and the fluid's vertical velocity is identical to the vertical motion of the grid. The z-tilde coordinate approaches a fully Lagrangian coordinate as $\tau_{D l f} \rightarrow \infty$.

\subsection{Baroclinic eddies test case}

The baroclinic eddies test case is the only one of the four idealized cases to use three dimensions and include the Coriolis force in the momentum equation. Horizontal mesh resolutions vary from $10 \mathrm{~km}$, which is eddy permitting, to $1 \mathrm{~km}$, where the large-scale structures of the eddies are well resolved, so this test case measures mixing under conditions closer to a realistic highresolution global ocean models than previous cases.

The domain is an idealization of the Antarctic Circumpolar Current. It is configured with periodic boundaries in $x$ and solid boundaries in $y$. Unlike similar idealized domains in the literature (Karsten et al., 2002; Hecht et al., 2008), this is a spin-down experiment with no surface wind or buoyancy forcing, so that the domain is a closed system. This ensures that RPE measures mixing without contamination by external influences.

The initial condition is stratified in the vertical, and shifts from warmer waters in the north to colder waters in the south within a narrow band, centered about a sine oscillation in the $x-y$ plane (Ilicak et al., 2012, Figure 15). The temperature distribution in the northern section is

$$
\Theta_{0}(z)=\Theta_{b o t}+\left(\Theta_{t o p}-\Theta_{b o t}\right) \frac{z_{b o t}-z}{z_{b o t}},
$$

where $\Theta_{\text {bot }}=10.1 \mathrm{C}, \Theta_{\text {top }}=13.1 \mathrm{C}$, and $z_{\text {bot }}=-975 \mathrm{~m}$ (note sign convention on $z$ ). The southern portion of the domain is uniformly $\Delta \Theta=1.2 \mathrm{C}$ cooler. 
The boundary between the northern and southern portions is defined by

$$
y_{w}(x)=y_{0}-y_{A} \sin \left(2 \pi k \frac{x}{L_{x}}\right)
$$

where $y_{0}=250 \mathrm{~km}, y_{A}=40 \mathrm{~km}, k=3$, and $L_{x}=160 \mathrm{~km}$, so that three wavelengths fill a domain of width $L_{x}$. The temperature distribution is then

$$
\Theta(x, y, z)= \begin{cases}\Theta_{0}(z) & y \geq y_{w}(x)+\Delta y \\ \Theta_{0}(z)-\Delta \Theta\left(1-\frac{y-y_{w}(x)}{\Delta y}\right) & y_{w}<y<y_{w}+\Delta y \\ \Theta_{0}(z)-\Delta \Theta & y \leq y_{w}(x)\end{cases}
$$

where the temperature is interpolated from north to south over a distance of $\Delta y=40 \mathrm{~km}$. An additional perturbation is added to one of the wave crests to incite the instability,

$$
\begin{aligned}
y_{w}^{\prime}(x) & =y_{0}-\frac{y_{A}}{2} \sin \left(\pi \frac{x-x_{2}}{x_{3}-x_{2}}\right) \\
\Theta^{\prime}(x, y) & =\Delta \Theta^{\prime}\left(1-\frac{y-y_{w}^{\prime}(x)}{\Delta y / 2}\right)
\end{aligned}
$$

so that the temperature is $\Theta+\Theta^{\prime}$ within the patch $x_{2} \leq x \leq x_{3}, y_{w}^{\prime}-\Delta y / 2 \leq$ $y \leq y_{w}^{\prime}+\Delta y / 2$, where $x_{2}=110 \mathrm{~km}, x_{3}=130 \mathrm{~km}$, and $\Delta \Theta^{\prime}=0.3 \mathrm{C}$.

A quadratic bottom drag is applied with a dimensionless coefficient of 0.01 . The simulation is conducted on a southern hemisphere f-plane with a Coriolis parameter of $-1.2 \times 10^{-4} \mathrm{~s}^{-1}$.

The tilted isopycnals are the source of potential energy for the baroclinic instability. Simulations are performed at resolutions with grid cell sizes of 1, 4 , and $10 \mathrm{~km}$ and horizontal viscosities ranging from 1 to $200 \mathrm{~m}^{2} \mathrm{~s}^{-1}$. At high viscosities (low Reynolds numbers) kinetic energy is low, eddies are large and smooth, and mixing is slower. Low viscosity simulations (higher Reynolds numbers) are more energetic, the eddies mix the background temperature gradient, and RPE increases more quickly. Higher horizontal resolution reduces the mixing rate because the grid can resolve larger gradients between cold and warm water, particularly around eddies. As time proceeds the initial distribution of tilted isopycnals flatten out, and the eddies spread to the boundaries (Figures 9 and 10). The rate of mixing slows significantly after 100 days when much of the initial temperature distribution has been smoothed (Figure 11). The simulations shown in Figure 12 were run with 
five values of viscosity $\left(\nu_{h}=1,5,10,20,200\right)$ for the $10 \mathrm{~km}$ and $4 \mathrm{~km}$ resolutions, and the lower four values for the $1 \mathrm{~km}$ resolution. For each viscosity, $d R P E / d t$ from POP z-level, POP z-star, MOM, and MITgcm are tightly clustered. MPAS-Ocean has lower mixing rates than the others; this is investigated further in the next section. Models vary in the southern-most y-extent of warm water at day 200, with MITgcm at $60 \mathrm{~km}$, ROMS between 0 and $50 \mathrm{~km}$ (Ilicak et al., 2012, Figure 16a,d), and MPAS-Ocean and POP at $0 \mathrm{~km}$ (Figures $9 \mathrm{~h}$ and 10b), where all of these images are for $\nu_{h}=20$ and $\Delta x=1 \mathrm{~km}$. Differences in y-extent of the warm water appear to be unrelated to differences in $d R P E / d t$, as POP and MITgcm have similar values in Figure 12 compared to MPAS-Ocean.

The baroclinic eddies test case is a good setting to compare z-level versus z-star in the same model, because it is three-dimensional, energetic, and runs for several hundred days. In z-star, the layer thickness is the same as in fixed z-levels, but with an additional small contribution to distribute SSH perturbations throughout the water column. These small changes in layer thickness do not reduce mixing in z-star. Indeed, $d R P E / d t$ results were nearly identical to z-level simulations for MPAS-Ocean in all cases (data not shown). Z-star is useful because the model may use extremely thin layers in the upper ocean. MPAS-Ocean has been successfully tested using z-star in realistic ocean domains with centimeter-thick layers. In addition, z-star can accommodate strong surface fluxes due to precipitation and evaporation without evacuating the top layer. The test cases and statistics presented here allow us to verify that z-star is working correctly. Figure 12 includes data from the POP ocean model with the traditional z-level configuration (Maltrud and McClean, 2005), and the newly implemented z-star formulation, which is detailed in Appendix A.

MPAS-Ocean tests using z-tilde on the baroclinic eddies case produce velocities that deform the grid as the fluid adjusts from large temperature gradients in $y$. This is similar to the application of z-tilde to the lock exchange, discussed in Section 3.2. The baroclinic eddies test case is not near equilibrium, and is not an appropriate application of z-tilde.

\subsection{Evaluation of Advection Methods and Cell Shape}

The rate of mixing is strongly sensitive to the tracer advection method. Here we use the $4 \mathrm{~km}$ resolution baroclinic eddies test case to evaluate mixing as a function of the order of the advection method. This is motivated by 
results in Figure 12, where MPAS-Ocean values for $d R P E / d t$ on a hexagon mesh are uniformly lower than POP, MOM, and MITgcm.

The tests were conducted within the framework of the flux-corrected transport (FCT) scheme, which is described for structured grids in Zalesak (1979) and Wang et al. (2009), and used in the development of transport schemes on unstructured grids in Skamarock and Gassmann (2011). The tracer flux at each cell edge, $F=u h \phi$, is composed of the velocity normal to the edge, $u$, the vertical thickness, $h$, and a value for the tracer, $\phi$, at that edge. In FCT, the flux

$$
F=F_{L}+r\left(F_{H}-F_{L}\right)
$$

is the sum of a low-order flux, $F_{L}$, and a flux correction term (Wang et al., 2009, eqn. 8). The weighting coefficient $r$ varies between 0 and 1 and is chosen to preserve monotonicity (i.e., no new minimums or maximums created in the tracer values). The low-order flux is chosen to be a monotonic method which, as a result, is diffusive; the high order flux, $F_{H}$, while preferred for its accuracy, is not a monotonic scheme. The standard FCT option in MPAS-Ocean uses a first-order upwind scheme for $F_{L}$ and a third-order reconstruction of $\phi$ to the edge for $F_{H}$ in both the horizontal and vertical. If monotonicity has been preserved by the high-order scheme, then the algorithm sets $r=1$ and only the high-order flux is used. Conversely, if $F_{H}$ violates monotonicity, $r$ is adjusted to add a portion of first-order upwind to the total flux. The scheme is formally first-order accurate due to the first-order contribution, but in practice produces smaller errors, as the leading-order truncation error term is much smaller than for second-order schemes (Skamarock and Gassmann, 2011).

Figure 13 shows a clear progression to lower $d R P E / d t$ values with higherorder advection. To vary the order of the FCT algorithm for this test, we set

$$
F_{H}=\alpha F_{1}+(1-\alpha) F_{2}
$$

where $F_{1}$ is the flux from first-order upwind advection, and $F_{2}$ uses secondorder tracer interpolation to the edge. The lowest order tested is pure first order, with $r=0$. All other tests use FCT, where $r$ is chosen by the algorithm at each edge to preserve monotonicity. As $\alpha$ is reduced from 1 to 0 , the high order flux is varied from first to second order, and $d R P E / d t$ is reduced in a regular fashion. Increasing from second to third-order tracer interpolation for $F_{H}$ produces nearly the same $d R P E / d t$ curve. 
In these tests, the tracer advection schemes for MITgcm was 7th-order monotonicity preserving advection; MOM used the multi-dimensional piecewise parabolic method (PPM), which is third order and includes a monotonicity constraint (Ilicak et al., 2012); and POP was run with 3rd-order upwind, which is not strictly monotonic. These three models produce nearly identical $d R P E / d t$ values, despite differences in advection methods and orders. MPAS-Ocean, with its standard FCT algorithm, is a factor of 5-10 lower in $d R P E / d t$, but can be brought to similar values by diminishing the order just slightly (by setting $\alpha=0.05$ ).

Our ability to increase mixing by decreasing the order of the advection method does not necessarily single out the FCT algorithm as the cause of lower spurious mixing in MPAS. After all, there are many differences amongst these models. MPAS-Ocean's hexagon horizontal grid cell shape is unique among these models and may be responsible for the reduced mixing; POP, MOM, and MITgcm all use quadrilateral grids. To test this hypothesis, the baroclinic eddies test case was repeated with MPAS-Ocean on a quadrilateral grid. The advection method is FCT with $F_{H}=F_{2}$. Mixing data from MPAS-Ocean on quadrilaterals is closer to the other quadrilateral models than MPAS-Ocean on hexagons (Figure 12), particularly at higher grid Reynolds numbers. Reduced spurious mixing on hexagon grids may be due to the representation of velocity on C-grid cells. Each cell expresses velocity as six vector components pointing in three directions (three unique degrees of freedom per cell), which is a more detailed representation of a continuous velocity field than four vector components pointing in two directions, as in a quadrilateral grid (two unique degrees of freedom per cell). Due to their geometry, hexagons pack $12-15 \%$ more grid cells into the same domain as quadrilaterals. This slight increase in resolution is not enough to explain the observed difference in $d R P E / d t$ between hexagon and quadrilateral grids.

\section{Global simulations}

We now proceed to the global real-world simulations. Two tests were conducted: a ten-year spin-up from rest to test the z-tilde scheme, and a ten-year spin-up followed by an unforced spin-down, in order to measure RPE and compare to Ilicak et al. (2012). All simulations use a z-star vertical coordinate, and some spin-up simulations use z-tilde in addition to z-star (see Section 2.1). The horizontal mesh is quasi-uniform over the globe, with simulations performed at nominal cell widths of 120,60, 30, and $15 \mathrm{~km}$. The mesh 
is based on Voronoi tessellations with uniform mesh density, which results in cells that are almost all hexagons. Initial distributions of potential temperature and salinity are obtained from the annual mean WOCE climatology (Gouretski and Koltermann, 2004). For the spin-up simulations, monthly mean wind stress is obtained from "Normal Year" forcing data from the Coordinated Ocean Reference Experiment (CORE, Large and Yeager (2004)), and surface temperature and salinity are restored to the initial condition with a time scale of 30 days, as in Ringler et al. (2013). Surface restoring is different from standard CORE experiments, which prescribe the atmospheric state and use bulk formulas (Griffies et al., 2009). Configuration settings similar to those presented in Ringler et al. (2013), and include the following: 40 vertical layers, ranging in thickness from $10 \mathrm{~m}$ near the surface to $250 \mathrm{~m}$ in the deep ocean; quadratic bottom drag with a coefficient of 0.001 ; nonlinear equation of state by Jackett and McDougall (1995); monotonic flux-corrected tracer transport (Skamarock and Gassmann, 2011), with third order reconstruction of the tracer values at the cell edges; split-explicit timestepping with 40 subcycles to step through $2 \Delta t$, and long baroclinic time steps $(\Delta t)$ of 3000,2400 , 1200, and 600 seconds for the 120,60,30, and $15 \mathrm{~km}$ resolutions, respectively. The horizontal turbulence closure is a simple biharmonic viscosity $\left(-\nabla^{4}\right)$ operator with coefficients of $\nu_{4 h}=\nu_{0}\left(\Delta x / \Delta x_{0}\right)^{3}$ where $\nu_{0}=5 \times 10^{10} \mathrm{~m}^{4} \mathrm{~s}^{-1}$ and $\Delta x_{0}=15 \mathrm{~km}$ cells. Zero horizontal diffusion is applied to the tracers. The vertical mixing is Richardson number-based, with background viscosity and diffusion of $10^{-4} \mathrm{~m}^{2} \mathrm{~s}^{-1}$ and $10^{-5} \mathrm{~m}^{2} \mathrm{~s}^{-1}$, respectively. Richardson number-based vertical tracer diffusion is on during the spin-up, and off for the spin-down. Constant vertical diffusion is a variable parameter for the spin-down simulations. See the MPAS-Ocean User's Guide (Jacobsen et al., 2013) for more details on how these settings are applied.

\subsection{Global spin-up}

The z-tilde scheme was tested with a global quasi-uniform resolution of $60 \mathrm{~km}$. Simulations begin from rest, and each layer has constant thickness in the horizontal. The filter time scale $\tau_{D l f}$ was varied from 0.1 to 10 days, and all cases used $\tau_{h h f}=5$ days and $\kappa_{h h f}=0$. Over the ten-year spin-up, average horizontal kinetic energy increases over the first five years and then levels off. All simulations have similar circulation patterns (not shown) and average kinetic energy within $7 \%$ of each other (Figure 14a).

The filter time scale is the main control on the frequency filtered coordinate system. When $\tau_{D l f}=0.1$ day, all baroclinic divergence is in the low 
frequency part, so the high frequency thickness is nearly zero. This simulation behaves the same way as when z-tilde is off (compare to z-level and z-star in Figure 14b). As the filter time scale increases, more thickness oscillations are included in the high frequency thickness. This reduces the vertical cross-coordinate transport (Figure 14b). Specifically, the time-averaged root mean squared vertical cross-coordinate transport is reduced from 2.3e$5 \mathrm{~m} / \mathrm{s}$ to $1.75 \mathrm{e}-5 \mathrm{~m} / \mathrm{s}$, a reduction of $24 \%$. Snapshots show that vertical cross-coordinate transport produced by internal waves along the equator is significantly reduced by the addition of z-tilde (Figure 15). Note that z-tilde does not suppress internal wave activity or vertical motion. It simply allows the grid to follow internal waves, or any fast vertical motion, in a Lagrangian fashion. These figures show that z-tilde reduces transport across grid cell interfaces in the vertical. Vertical motion of the fluid is not suppressed, as it is the sum of cross-coordinate transport and grid motion. Z-tilde results in the reduction of unphysical mixing caused by internal waves, as shown in the internal wave test case (Section 3.4).

The extra computation required for the frequency filtered coordinate system is in solving the two additional prognostic equations (12 and 13), adding high frequency thickness to the desired ALE thickness, and then altering the desired thickness to be within minimum and maximum constraints (7). Compute times for the forward model increased by $4 \%$ per time step for the global $60 \mathrm{~km}$ mesh. Timing excludes i/o and analysis calculations, and was tested on typical configurations of 512 processors. A larger problem is that the addition of frequency-filtering often imposes a stricter time step for stability, usually half the standard time step when $\tau_{D l f}=5$ days and a quarter when $\tau_{D l f}=10$ days. This is a significant hindrance to the use of frequency-filtered vertical coordinates, and a problem that we are working to improve.

\subsection{Global spin-down}

The second set of global simulations begin after year ten of the spinup simulations in the previous section. Wind forcing and temperature and salinity restoring are turned off, and the simulations are continued for 70 days to assess the mixing characteristics of the model. The turbulence closure for the momentum equation remains the same as the forced case, and may vary amongst models. MPAS-Ocean used biharmonic viscosity in the horizontal and Richardson-number based momentum mixing in the vertical. The POP turbulence closures for momentum included anisotropic horizontal viscosity with standard settings, KPP (Large et al., 1994) in the vertical for spin-up, 
and constant $\left(\nu_{v}=2.5 \mathrm{e}-5 \mathrm{~m}^{2} \mathrm{~s}^{-1}\right)$ for spin-down. POP used Gent-McWilliams (Gent and McWilliams, 1990) as a tracer turbulence closure for spin-up. For all models, tracer diffusion during spin-down is zero in the horizontal and constant in the vertical, with a coefficient varying from $\kappa_{v}=0$ to $\kappa_{v}=10^{-5}$ $\mathrm{m}^{2} \mathrm{~s}^{-1}$ (Ilicak et al., 2012).

Mixing is a strong function of vertical tracer diffusion; for all models, $d R P E / d t$ increases with $\kappa_{v}$ at a fixed resolution (Figure 16). At lower resolutions, POP displays the highest mixing, MOM the least, and MPAS-Ocean is between the two. Both POP and MOM use nominally one-degree quadrilateral grids with typical horizontal grid length of $100 \mathrm{~km}$. For a particular $\kappa_{v}$ all the low-resolution simulations report $d R P E / d t$ values within a factor of two, and so appear to be in good agreement overall.

As one proceeds to higher resolution, MPAS-Ocean simulations have less mixing. This is similar to the behavior of the baroclinic eddy case at a fixed horizontal viscosity (Figure 12). Ilicak et al. (2012) found the opposite: their MOM $1 / 4^{\circ}$ spin-down produced higher $d R P E / d t$ than the one-degree case due to mixing by the eddies. In order to better understand these differences, we conducted a sensitivity study of $d R P E / d t$ versus grid Reynolds number (Figure 17). In a typical series of simulations with varying resolution, the biharmonic viscosity is chosen to scale with $(\Delta x)^{3}$, so that the grid Reynolds number is nearly constant (see equation 19). This is the case for the MPASOcean simulations in Figure 16 with $\kappa_{v}=0$, which appear as the middle points in Figure 17, with grid Reynolds numbers between 5 and 10. (Small differences between these two series are due to monthly versus annual mean forcing during the spin-up in Figures 16 and 17, respectively.) The results in Figure 17 all used $\kappa_{v}=0$, were spun-up for ten years with a standard $\nu_{h}$ value for each resolution, and then continued for one additional year of spinup with the tested $\nu_{h}$ values before the 70 day spin-down experiment. At the lowest grid Reynolds number, the fluid is sufficiently viscous that spurios mixing, as measured by $d R P E / d t$, is not resolution dependant. For more typical values of viscosity, the spurious mixing decreases monotonically with increasing resolution at a fixed grid Reynolds number. With this information, a user may choose the value of numerical mixing they are willing to live with, and then read off the required resolution for a given grid Reynolds number.

The addition of z-tilde did not cause any appreciable reduction in $d R P E / d t$ in the global spin-down experiments. The z-tilde coordinate is only appropriate for well-equilibriated simulations. The spin-down simulations begin by turning off wind forcing and surface restoring at year ten of spun-up simula- 
tions, causing large transient flows to readjust to the new forcing conditions. As shown with the lock exchange test case (Section 3.2), transient flows that occur at similar or faster time-scales than the frequency filter $\left(\tau_{D l f}\right)$ result in simulations with similar or higher values of $d R P E / d t$.

\section{Conclusions}

In the development of a new ocean-climate model, verification of model behavior against observations and standard test cases is a critical part of the development process. Published results of model intercomparisons give model developers and users confidence in the proper functioning of the model, and an understanding of what model components need further improvement. The best place to start is comparison of idealized test cases with simple initial conditions and very few parameters, so that individual processes may be isolated and assessed. In the end, an ocean-climate model must be evaluated in real-world simulations if it is to fulfill its goal of decadal and centurylong climate predictions. In this work we evaluate the vertical coordinate of MPAS-Ocean using numerous configurations ranging from idealized to realistic.

The overall conclusion of these tests is that the MPAS-Ocean ALE vertical coordinate can correctly represent z-level, z-star (with and without partial bottom cells), z-tilde, isopycnal and sigma coordinates with mixing rates, as measured by $d R P E / d t$, that are commensurate with existing ocean models. In addition, a newly implemented z-star coordinate in POP was shown to produce nearly identical results as the standard z-level formulation, as expected. The test problems fully specify the domain, initial conditions, viscosity, and diffusion, so differences in mixing rates must be due to model numerics such as the horizontal and vertical coordinates, discretization methods, derivative operators, time-stepping schemes, and advection schemes.

MPAS-Ocean has much lower spurious mixing than other models in threedimensional domains, which are the baroclinic eddies and high-resolution global spin-down cases. This result, while encouraging, must be thoroughly vetted to be trusted and understood. Two features of MPAS-Ocean stand out as different from the other ocean models tested: a horizontal grid based on Voronoi tesselations (mostly hexagons), and the flux-corrected transport (FCT) tracer advection scheme with interpolations designed for unstructured grids. MPAS-Ocean simulations carried out on hexagon and quadrilateral grids show that spurious mixing is lower on hexagon grids, particularly at 
high grid Reynolds numbers. Another set of simulations tested the tracer advection algorithm, and showed that a small reduction in the order of accuracy produces $d R P E / d t$ values similar to the other models. In addition, identical domain set-up and analysis tools used for MPAS-Ocean were used for POP in the baroclinic eddies and global spin-down cases; the POP results were nearly identical to MOM and MITgcm. We conclude that both the advection scheme and hexagon-type grids play a role in the reduced spurious mixing by MPAS-Ocean. In two-dimensional $(x-z)$ domains MPAS-Ocean produced spurious mixing similar to or higher than other models, which indicates that the advection schemes and horizontal grid type plays a stronger role in reducing mixing in fully three-dimensional domains.

The frequency-filtered scheme (z-tilde) reduces vertical transport across layer interfaces. This occurs in a consistent way as a function of the filter time scale: in the internal wave test case by over a factor of ten, and the global spin-up case by $24 \%$. This leads to a reduction in spurious mixing caused by internal waves. The z-tilde scheme requires solving two additional prognostic equations, which adds $4 \%$ to the compute time of each time step. In the internal gravity wave test case the standard time step could be used, but in global simulations with z-tilde the time step had to be reduced to half its standard value when $\tau_{D l f}=5$ days. We hope to improve the time step, but this issue could be a hindrance for z-tilde in practical applications.

It was shown that z-tilde is not appropriate in simulations with large, fast transient flows, such as the lock exchange, overflow, and baroclinic eddies test cases, and the global spin-down. In these cases the grid follows the fast flows in a Lagrangian manner, but becomes too distorted, so that mixing is not reduced from a z-level or z-star coordinate. The z-tilde scheme is effective at reducing mixing for small, fast perturbations, such as those caused by internal gravity waves.

Development of MPAS-Ocean began in 2010, and progressed to its first public release in 2013. The comparisons against long-standing ocean models presented here have been an intrinsic part of model development, verification, and improvement. In particular, these results show that the MPAS-Ocean vertical coordinate is robust, and produces spurious mixing that is similar to or less than other ocean models. 


\section{Acknowledgement}

The authors thank A. Adcroft and an anonymous reviewer for helpful feedback, and M. Ilicak for providing useful feedback, data in electronic form, and details of the initial conditions for the four test cases. Simulations were conducted using LANL Institutional Computing resources. This work was supported by the Earth System Modeling and Regional and Global Climate Modeling programs of the Office of Biological and Environmental Research within the US Department of Energy's Office of Science.

\section{Appendix A. The POP z-star formulation}

The new POP vertical coordinate may be run in z-level or z-star mode. It is not a full ALE implementation, which requires a prognostic thickness equation, as in (2), but diagnoses the thickness based on the sea surface height (SSH),

$$
h_{k}=d z_{k}\left(1+\eta \frac{W_{k}}{\sum_{k^{\prime}=1}^{k m a x} d z_{k^{\prime}} W_{k^{\prime}}}\right),
$$

where the weights $W_{k}$ are described in Table $3, \eta$ is the $\mathrm{SSH}$, and $d z_{k}$ is the original thickness of the z-level model.

Here we refer to the POP reference manual (Smith et al., 2010), hereafter POP. The z-level form of the momentum equation requires the addition of the $\nabla z_{\text {mid }}$ term for tilted layers, shown in (1). This has the form

$$
\left(-\frac{\rho g}{\rho_{0}} \delta_{x} \bar{z}_{m i d}^{y},-\frac{\rho g}{\rho_{0}} \delta_{y} \bar{z}_{m i d}^{x}\right),
$$

added to the right-hand sides of POP eqns 3.21 and 3.22 .

The tracer equation becomes thickness weighted, so that POP eqn 3.11 is now

$$
\frac{\partial}{\partial t} h \varphi+d z \mathcal{L}_{T}(\varphi)=d z \mathcal{D}_{H}(\varphi)+d z \mathcal{D}_{V}(\varphi)+d z \mathcal{F}_{W}(\varphi),
$$

where the tracer advection, horizontal diffusion, vertical diffusion, and freshwater input remain unchanged (POP eqns 3.13, 3.14, 3.18, 3.19). 
In the time stepping, the barotropic step remains untouched. The baroclinic time-stepping will change by factors of thickness in many places. Previously in z-level coordinates, a scaling factor was included to account for $\mathrm{SSH}$ in the top layer only (POP eqn 3.12),

$$
\xi=\frac{\delta_{k 1}}{d z_{1}} \eta
$$

The tracer equation is now thickness-weighted, so that the factor $\left(1+\xi^{n}\right)$ may be replaced by $h^{n}$ in the predictor/corrector time stepping scheme, and likewise for $n+1$ and $n-1$ (POP equations 4.1-4.15).

\section{References}

Adcroft, A., Campin, J.M., 2004. Rescaled height coordinates for accurate representation of free-surface flows in ocean circulation models. Ocean Modelling 7, 269-284. doi:10.1016/j.ocemod.2003.09.003.

Adcroft, A., Hallberg, R., 2006. On methods for solving the oceanic equations of motion in generalized vertical coordinates. Ocean Modelling 11, 224233. doi:10.1016/j.ocemod.2004.12.007.

Adcroft, A., Hill, C., Marshall, J., 1997. Representation of Topography by Shaved Cells in a Height Coordinate Ocean Model. Monthly Weather Review 125, 2293. doi:10.1175/1520-0493(1997)125¡2293:ROTBSC ¿2.0.CO;2.

Alford, M.H., 2003. Redistribution of energy available for ocean mixing by long-range propagation of internal waves. Nature 423, 159-162.

Benjamin, T.B., 1968. Gravity currents and related phenomena. J. Fluid Mech. 31, 209-248. doi:10.1017/S0022112068000133.

Bleck, R., 1978. On the Use of Hybrid Vertical Coordinates in Numerical Weather Prediction Models. Monthly Weather Review 106, 1233. doi:10.1175/1520-0493(1978)106¡1233:OTUOHV ¿2.0.CO;2.

Bleck, R., 2002. An oceanic general circulation model framed in hybrid isopycnic-Cartesian coordinates. Ocean Modelling 4, 55-88. doi:10.1016/S1463-5003(01)00012-9. 
Bryan, K., Manabe, S., Pacanowski, R.C., 1975. A Global Ocean-Atmosphere Climate Model. Part II. The Oceanic Circulation. J. Phys. Oceanography 5, 30-46. doi:10.1175/1520-0485(1975)005;0030:AGOACMi2.0.CO;2.

Donea, J., Huerta, A., Ponthot, J.P., Rodrguez-Ferran, A., 2004. Arbitrary LagrangianEulerian Methods, in: Encyclopedia of Computational Mechanics.. John Wiley \& Sons, Ltd, New York, pp. 1-25.

Fringer, O.B., Gerritsen, M., Street, R.L., 2006. An unstructured-grid, finitevolume, nonhydrostatic, parallel coastal ocean simulator. Ocean Modelling 14, 139-173. doi:10.1016/j.ocemod.2006.03.006.

Gent, P.R., McWilliams, J.C., 1990. Isopycnal mixing in ocean circulation models. J. Phys. Oceanography 20, 150-155.

Gouillon, F., 2010. Internal wave propagation and numerically induced diapycnal mixing in oceanic general circulation models. Ph.D. thesis. Florida State University.

Gouretski, V., Koltermann, K.P., 2004. WOCE global hydrographic climatology. Berichte des BSH 35, 1-52.

Griffies, S., 2004. Fundamentals of Ocean Climate Models. first ed., Princeton University Press, Princeton, USA.

Griffies, S.M., Biastoch, A., Böning, C., Bryan, F., Danabasoglu, G., Chassignet, E.P., England, M.H., Gerdes, R., Haak, H., Hallberg, R.W., Hazeleger, W., Jungclaus, J., Large, W.G., Madec, G., Pirani, A., Samuels, B.L., Scheinert, M., Gupta, A.S., Severijns, C.A., Simmons, H.L., Treguier, A.M., Winton, M., Yeager, S., Yin, J., 2009. Coordinated Ocean-ice Reference Experiments (COREs). Ocean Modelling 26, 1-46. doi:10.1016/j.ocemod.2008.08.007.

Griffies, S.M., Gnanadesikan, A., Dixon, K.W., Dunne, J.P., Gerdes, R., Harrison, M.J., Rosati, A., Russell, J.L., Samuels, B.L., Spelman, M.J., Winton, M., Zhang, R., 2005. Formulation of an ocean model for global climate simulations. Ocean Science 1, 45-79.

Griffies, S.M., Hallberg, R.W., 2000. Biharmonic Friction with a Smagorinsky-Like Viscosity for Use in Large-Scale Eddy-Permitting Ocean 
Models. Monthly Weather Review 128, 2935. doi:10.1175/15200493(2000)128;2935:BFWASLi2.0.CO;2.

Hecht, M.W., Holm, D.D., Petersen, M.R., Wingate, B.A., 2008. Implementation of the LANS-alpha turbulence model in a primitive equation ocean model. J. Comp. Phys. 227, 5691-5716.

Hirt, C.W., Amsden, A.A., Cook, J.L., 1974. An arbitrary LagrangianEulerian computing method for all flow speeds. J. Comp. Phys. 14, 227253.

Ilicak, M., Adcroft, A.J., Griffies, S.M., Hallberg, R.W., 2012. Spurious dianeutral mixing and the role of momentum closure. Ocean Modelling 45, 37-58. doi:10.1016/j.ocemod.2011.10.003.

Jackett, D.R., McDougall, T.J., 1995. Minimal Adjustment of Hydrographic Profiles to Achieve Static Stability. Journal of Atmospheric and Oceanic Technology 12, 381. doi:10.1175/15200426(1995)012;0381:MAOHPT¿2.0.CO;2.

Jacobsen, D., Petersen, M., Ringler, T., 2013. MPAS-Ocean model user's guide. Technical Report. Los Alamos National Laboratory. URL: http://mpas-dev.github.io.

Karsten, R., Jones, H., Marshall, J., 2002. The role of eddy transfer in setting the stratification and transport of a circumpolar current. J. Phys. Oceanography $32,39-54$.

Large, W.G., McWilliams, J.C., Doney, S.C., 1994. Oceanic vertical mixing: a review and a model with a nonlocal boundary layer parameterization. Rev. Geophys. 32, 363-404.

Large, W.G., Yeager, S., 2004. Diurnal to Decadal Global Forcing For Ocean and Sea-Ice Models:The Data Sets and Flux Climatologies.

Leclair, M., Madec, G., 2011. Z-tilde Coordinate, an Arbitrary LagrangianEulerian coordinate separating high and low frequency motions. Ocean Modelling 37, 139-152. doi:10.1016/j.ocemod.2011.02.001.

Legg, S., Hallberg, R.W., Girton, J.B., 2006. Comparison of entrainment in overflows simulated by z-coordinate, isopycnal and non-hydrostatic models. Ocean Modelling 11, 69-97. 
Maltrud, M.E., McClean, J.L., 2005. An eddy resolving global 1/10^o ocean simulation. Ocean Modelling 8, 31-54. doi:10.1016/j.ocemod.2003.12.001.

Pacanowski, R.C., Gnanadesikan, A., 1998. Transient Response in a Z-Level Ocean Model That Resolves Topography with Partial Cells. Monthly Weather Review 126, 3248. doi:10.1175/15200493(1998)126;3248:TRIAZL ¿2.0.CO;2.

Ringler, T., Gent, P., 2011. An eddy closure for potential vorticity. Ocean Modelling 39, 125-134. doi:10.1016/j.ocemod.2011.02.003.

Ringler, T., Ju, L., Gunzburger, M., 2008. A multiresolution method for climate system modeling: application of spherical centroidal Voronoi tessellations. Ocean Dynamics 58, 475-498. doi:10.1007/s10236-008-0157-2.

Ringler, T., Petersen, M., Higdon, R.L., Jacobsen, D., Jones, P.W., Maltrud, M., 2013. A multi-resolution approach to global ocean modeling. Ocean Modelling 69, 211-232. doi:10.1016/j.ocemod.2013.04.010.

Ringler, T.D., Thuburn, J., Klemp, J.B., Skamarock, W.C., 2010. A unified approach to energy conservation and potential vorticity dynamics for arbitrarily-structured C-grids. J. Comp. Physics 229, 3065-3090. doi:10.1016/j.jcp.2009.12.007.

Saenz, J.A., Tailleux, R., Butler, E., Hughes, G., Oliver, K., 2014. Estimating Lorenz's reference state in an ocean with a non-linear equation of state for seawater. In preparation.

Shchepetkin, A.F., McWilliams, J.C., 2003. A method for computing horizontal pressure-gradient force in an oceanic model with a nonaligned vertical coordinate. Journal of Geophysical Research (Oceans) 108, 3090. doi:10.1029/2001JC001047.

Shchepetkin, A.F., McWilliams, J.C., 2005. The regional oceanic modeling system (ROMS): a split-explicit, free-surface, topographyfollowing-coordinate oceanic model. Ocean Modelling 9, 347-404. doi:10.1016/j.ocemod.2004.08.002.

Shchepetkin, A.F., McWilliams, J.C., 2011. Accurate Boussinesq oceanic modeling with a practical, stiffened Equation of State. Ocean Modelling 38, 41-70. doi:10.1016/j.ocemod.2011.01.010. 
Skamarock, W.C., Gassmann, A., 2011. Conservative Transport Schemes for Spherical Geodesic Grids: High-Order Flux Operators for ODEBased Time Integration. Monthly Weather Review 139, 2962-2975. doi:10.1175/MWR-D-10-05056.1.

Skamarock, W.C., Klemp, J.B., Duda, M.G., Fowler, L.D., Park, S.H., Ringler, T.D., 2012. A Multiscale Nonhydrostatic Atmospheric Model Using Centroidal Voronoi Tesselations and C-Grid Staggering. Monthly Weather Review 140, 3090-3105. doi:10.1175/MWR-D-11-00215.1.

Smith, R., Jones, P., et al., 2010. The Parallel Ocean Program (POP) Reference Manual Ocean Component of the Community Climate System Model (CCSM) and Community Earth System Model (CESM). Technical Report. Los Alamos National Laboratory. LAUR-10-01853.

Smith, R.D., Dukowicz, J.K., Malone, R.C., 1992. Parallel ocean general circulation modeling. Physica D 60, 38-61.

Song, Y.T., 1998. A General Pressure Gradient Formulation for Ocean Models. Part I: Scheme Design and Diagnostic Analysis. Monthly Weather Review 126, 3213. doi:10.1175/1520-0493(1998)126;3213:AGPGFF ¿2.0.CO;2.

Thuburn, J., Ringler, T.D., Skamarock, W.C., Klemp, J.B., 2009. Numerical representation of geostrophic modes on arbitrarily structured C-grids. Journal of Computational Physics 228, 8321-8335. doi:10.1016/j.jcp.2009.08.006.

Wang, H., Skamarock, W.C., Feingold, G., 2009. Evaluation of Scalar Advection Schemes in the Advanced Research WRF Model Using Large-Eddy Simulations of Aerosol-Cloud Interactions. Monthly Weather Review 137, 2547. doi:10.1175/2009MWR2820.1.

Willebrand, J., 2001. Circulation characteristics in three eddy-permitting models of the North Atlantic. Progress in Oceanography 48, 123-161. doi:10.1016/S0079-6611(01)00003-9.

Zalesak, S.T., 1979. Fully multidimensional flux-corrected transport algorithms for fluids. Journal of Computational Physics 31, 335-362. doi:10.1016/0021-9991(79)90051-2. 

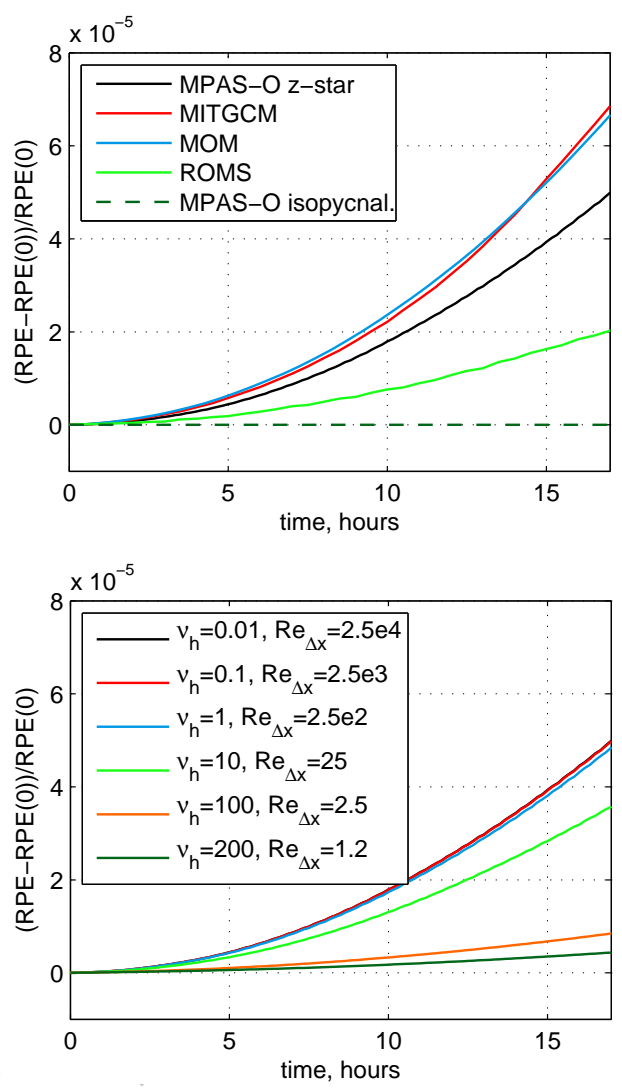

(b)

(c)

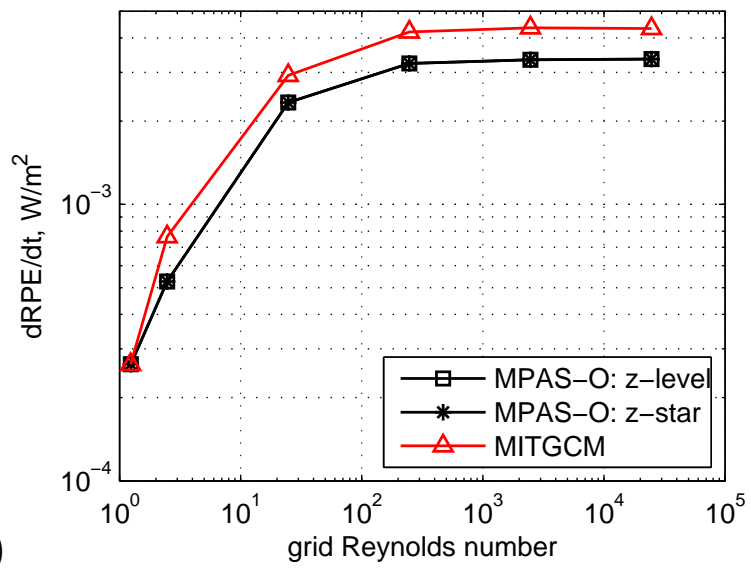

Figure 2: Lock exchange test case: Normalized resting potential energy (a) for several models using a horizontal viscosity $\nu_{h}=0.01$ and (b) for MPAS-Ocean using z-star and varying horizontal viscosity; (c) rate of change of RPE at 17 hours using the $\nu_{h}$ values in (b). 


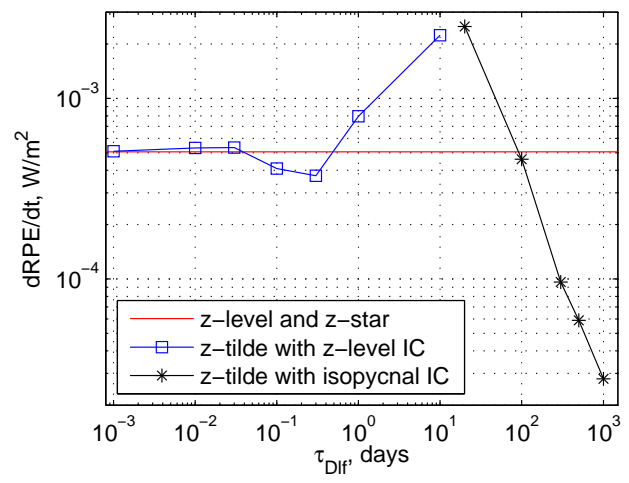

Figure 3: Lock exchange test case: Rate of change of RPE at 17 hours as a function of the frequency filter time scale, $\tau_{D l f}$, using the z-tilde vertical coordinate. These simulations used values of $\nu_{h}=100 \mathrm{~m}^{2} / \mathrm{s}$, and $\kappa_{h h f}=0$. The $d R P E / d t$ value for z-level and z-star are identical (red line), and are not a function of $\tau_{D l f}$. The two series begin with z-level (blue) or isopycnal (black) initial conditions (IC), and use $\tau_{h h f}=30$ days and $\tau_{h h f}=0$, respectively. 

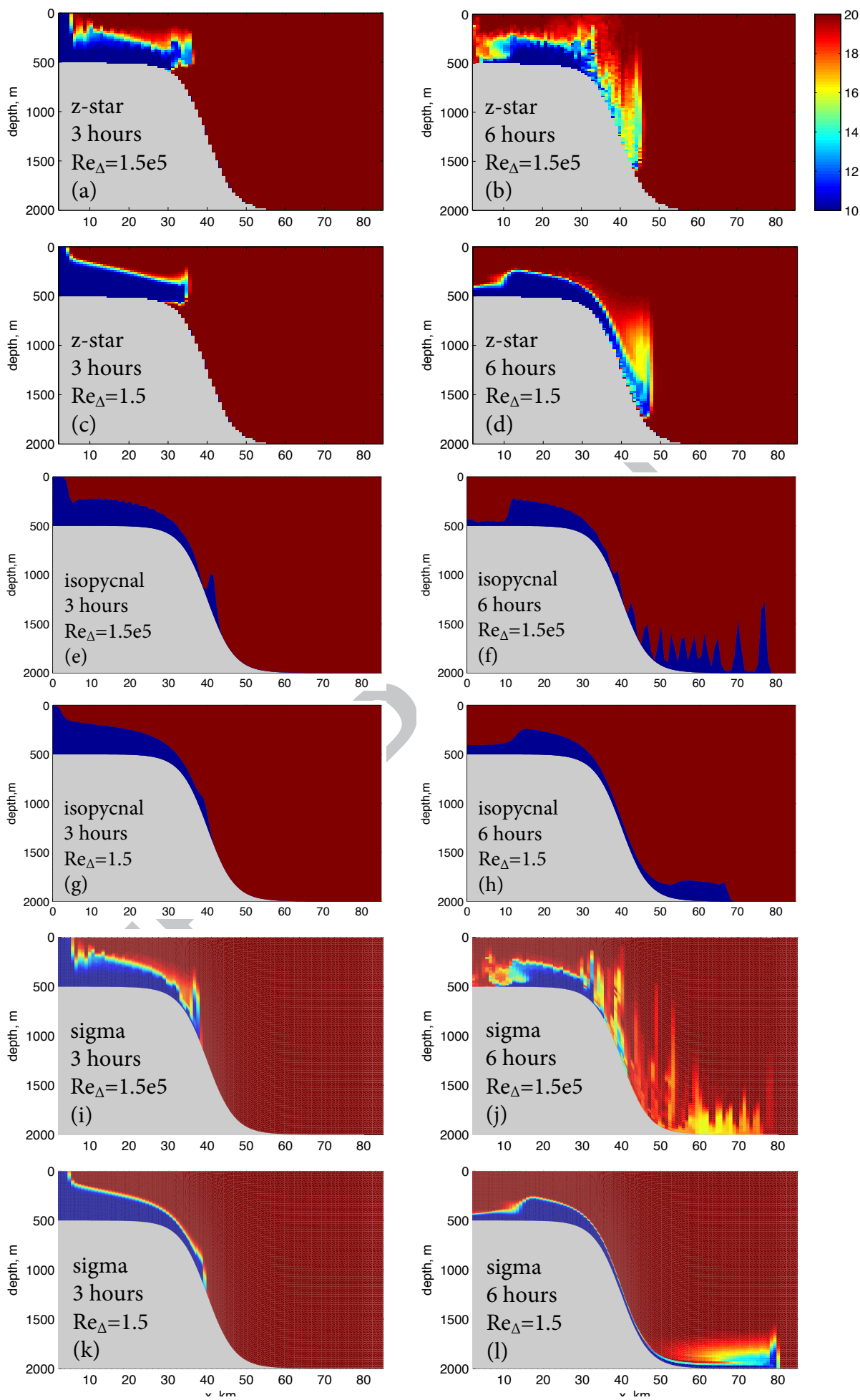

41

Figure 4: Overflow test case: Images from MPAS-Ocean at three hours (left column) and six hours (right column) for z-star (a-d), isopycnal (e-h) and sigma vertical coordinates (i-l). Two cases of horizontal viscosity are shown, $\nu_{h}=10^{-2}$ and $\nu_{h}=10^{3} \mathrm{~m}^{2} \mathrm{~s}^{-1}$, corresponding to grid Reynolds numbers of $R e_{\Delta}=1.5 \mathrm{e} 5$ and $R e_{\Delta}=1.5$. 


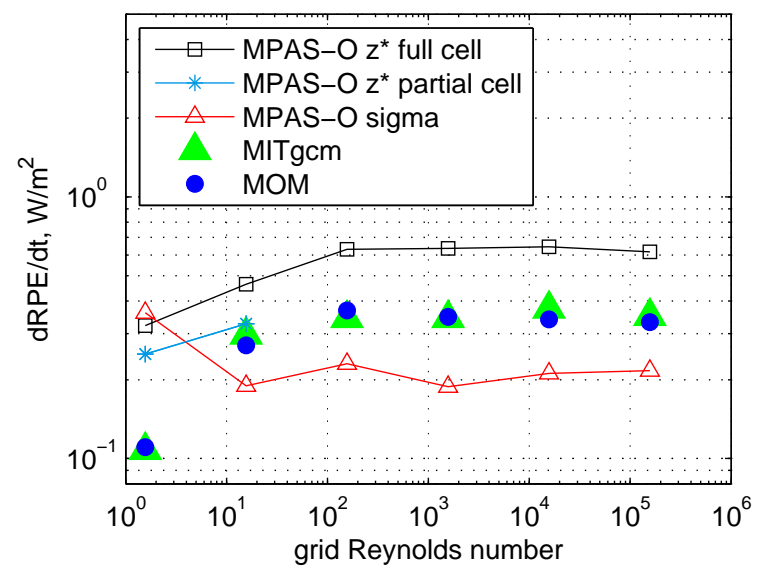

Figure 5: Overflow test case: Rate of change of RPE, measured from 13-37 hours. MPASOcean z-level results (not shown) are identical to the z-star results. 

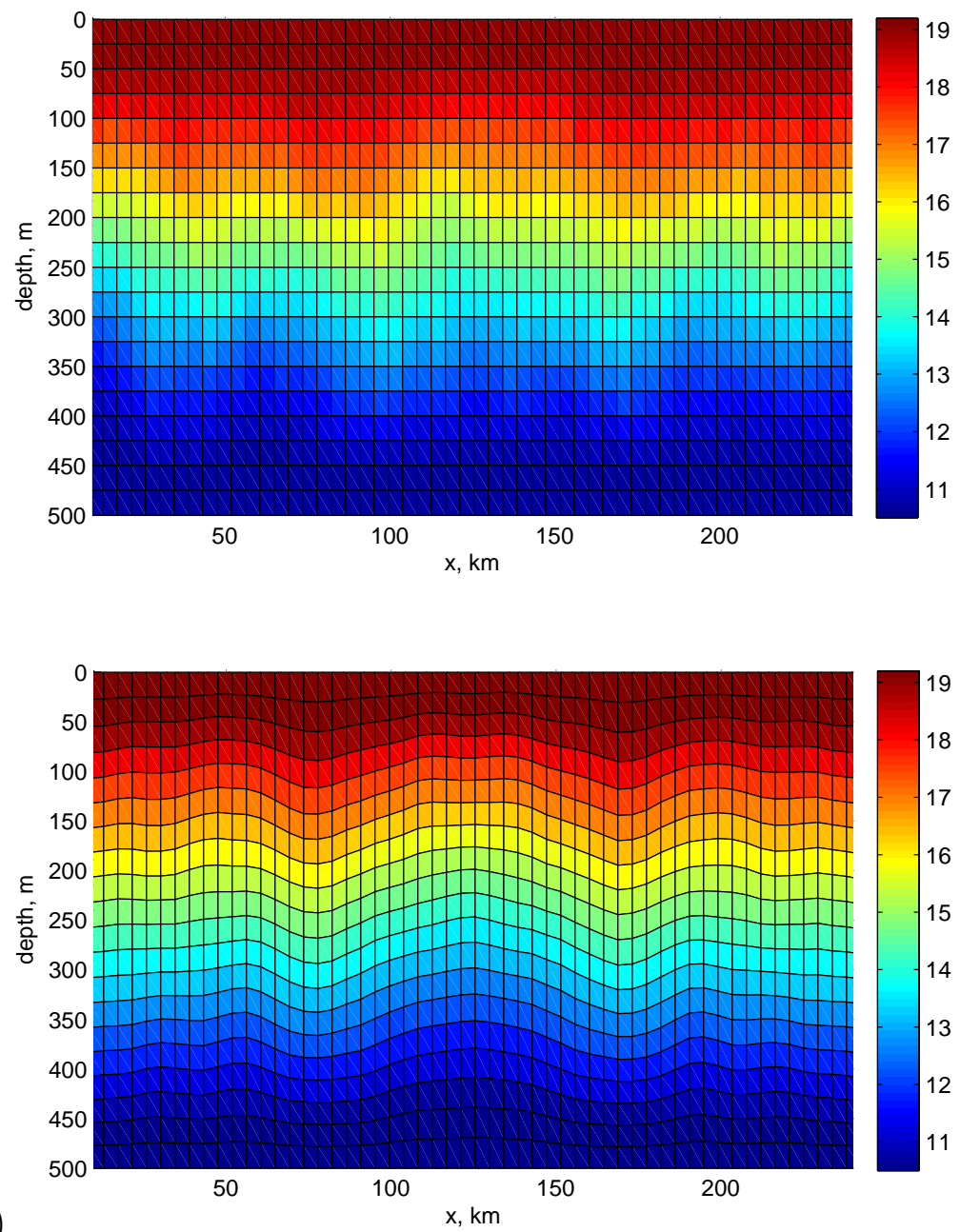

Figure 6: Internal wave test case: Temperature at 200 days with $\nu_{h}=0.01$ and $R e_{\Delta}=5 \mathrm{e} 4$, using (a) z-star and (b) z-tilde with $\tau_{D l f}=10$ days. Black lines show positions of grid cell boundaries. 


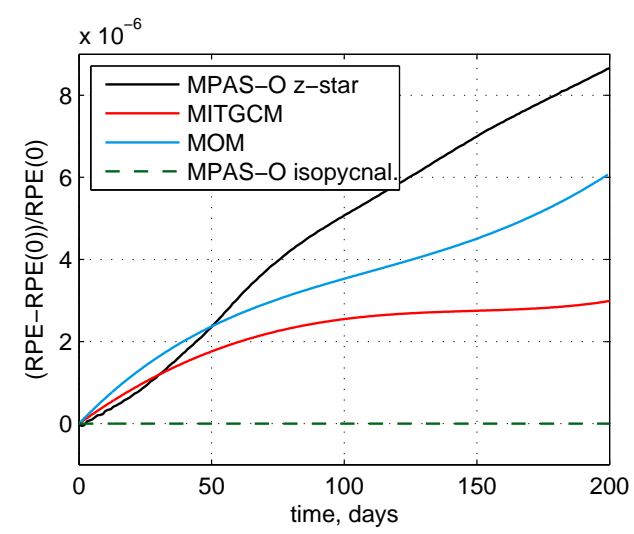

(b)
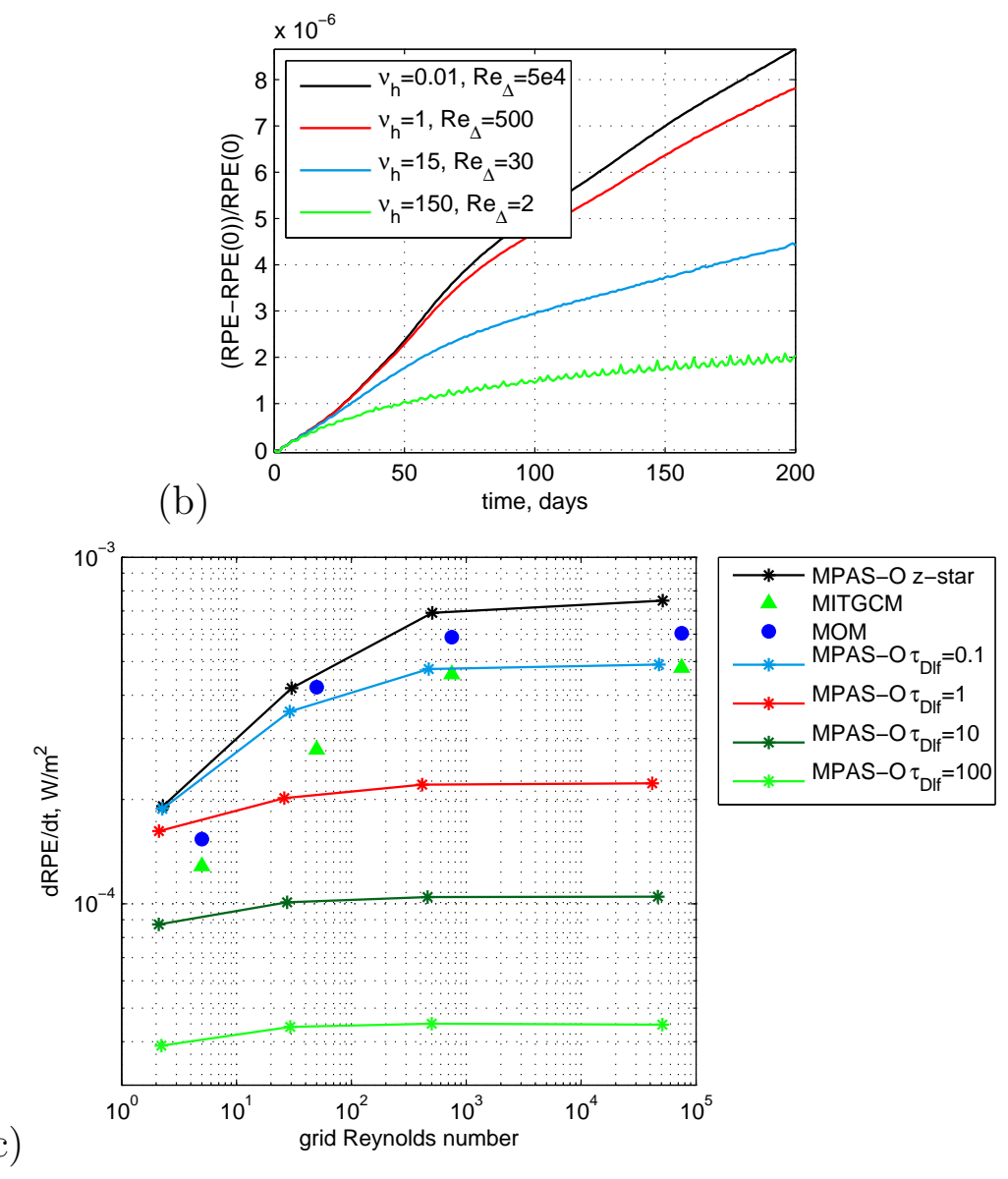

Figure 7: Internal wave test case: Normalized RPE (a) using several models with horizontal viscosity $\nu_{h}=0.01$, and (b) using MPAS-Ocean with z-star and varying horizontal viscosities; (c) rate of change of RPE, averaged from day 10 to 100, using the $\nu_{h}$ values in (b). Legend entries with $\tau_{D l f}$ in (c) indicate the z-tilde coordinate. As the filter time scale $\left(\tau_{D l f}\right)$ is increased from 0.1 to 100 days, $d R P E / d t$ decreases, showing reduced vertical mixing. Z-level results overlay those for z-stat, and are not shown. 

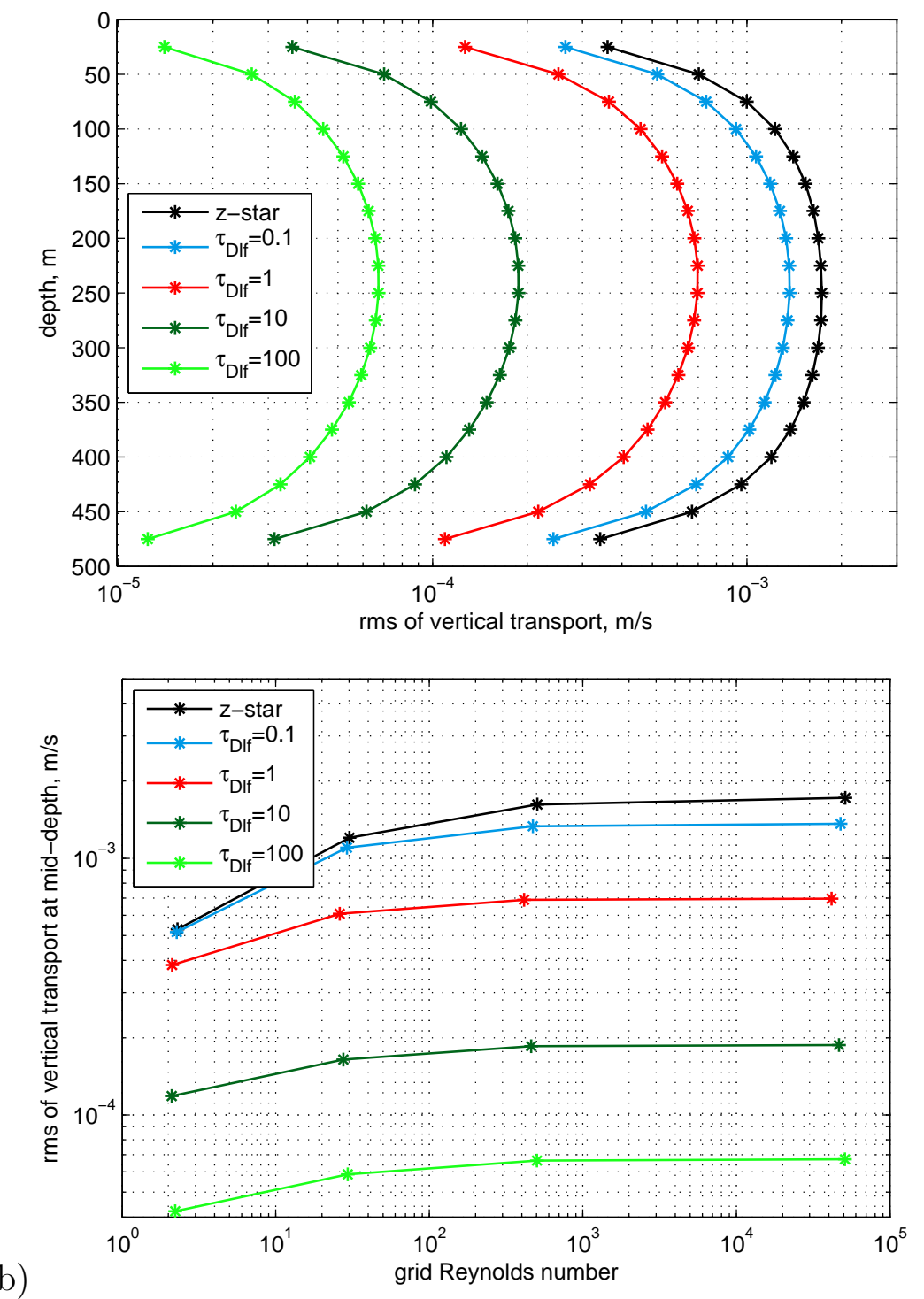

Figure 8: Internal wave test case: RMS of vertical cross-coordinate transport at the layer interface averaged from day 10 to 100 , using MPAS-Ocean. As the filter time scale $\left(\tau_{D l f}\right)$ is increased from 0.1 to 100 days, the vertical cross-coordinate transport between layers decreases. The top panel (a) shows the horizontal average for simulations with $\nu_{h}=0.01$, and lower panel (b) shows domain average for $\nu_{v}=0.01,1,15,150$. Legend entries with $\tau_{D l f}$ indicate the z-tilde coordinate. 


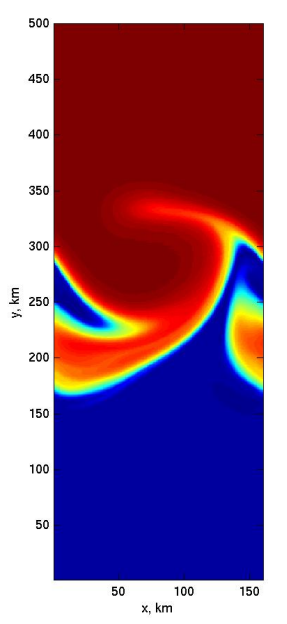

(a) day $50 \nu_{h}=200$

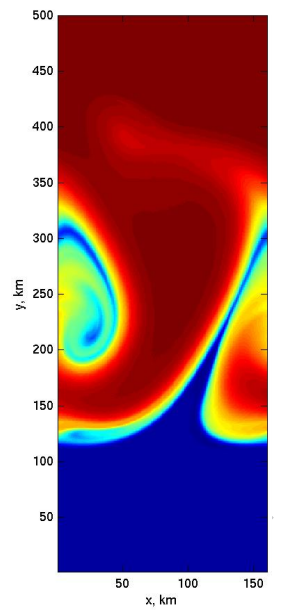

(d) day $100 \nu_{h}=200$

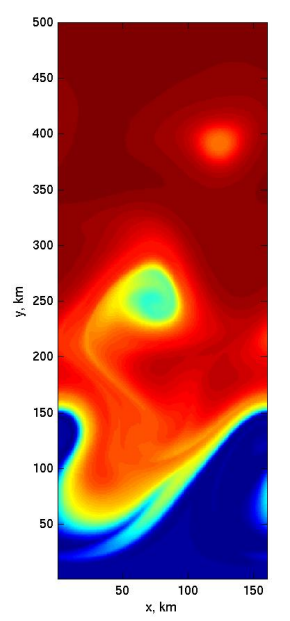

(g) day $200 \nu_{h}=200$

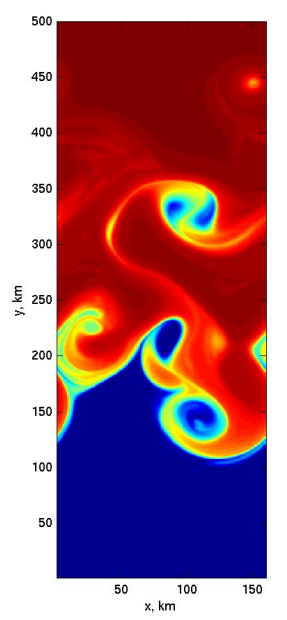

(b) day $50 \nu_{h}=20$

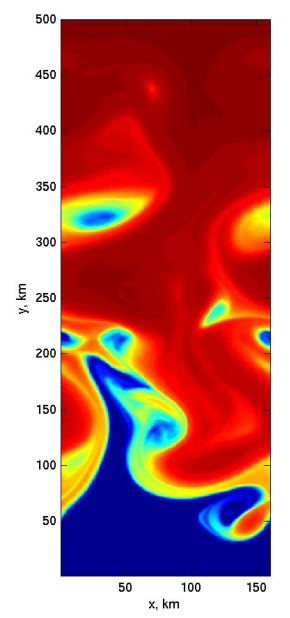

(e) day $100 \nu_{h}=20$

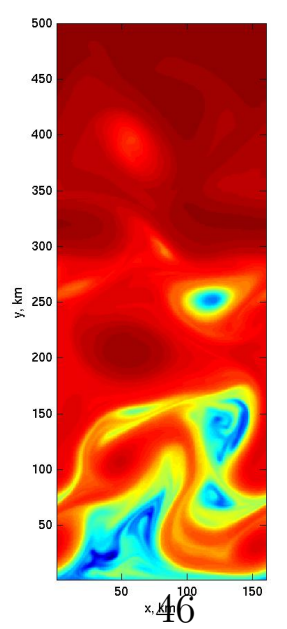

(h) day $200 \nu_{h}=20$

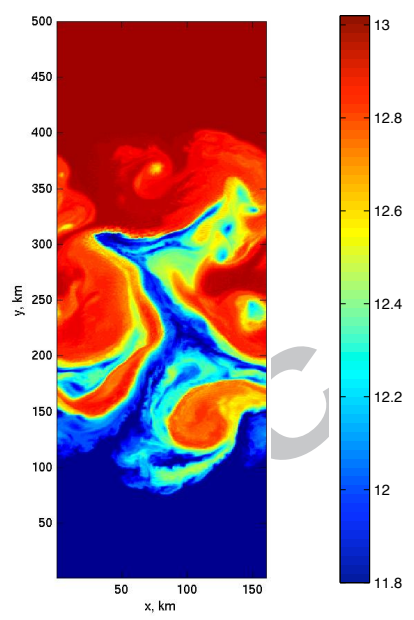

(c) day $50 \nu_{h}=1$

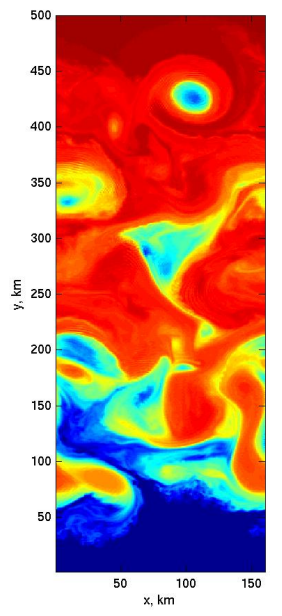

(f) day $100 \nu_{h}=1$

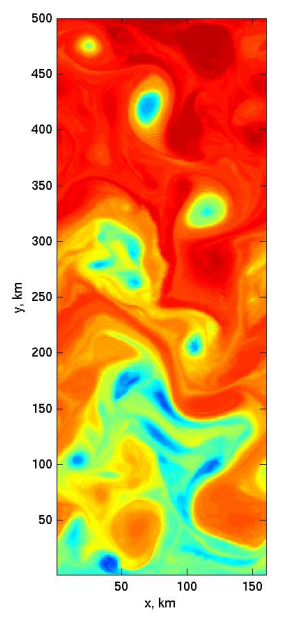

(i) day $200 \nu_{h}=1$

Figure 9: Baroclinic eddies test case: MPAS-Ocean with z-star, temperature, top layer $(\mathrm{k}=1)$, using a resolution of $\Delta x=1 \mathrm{~km}$. Horizontal viscosity values of $\nu_{h}=200,20$, and 1 are associated with grid Reynolds numbers of $R e_{\Delta}=0.3,4$, and 100 . 


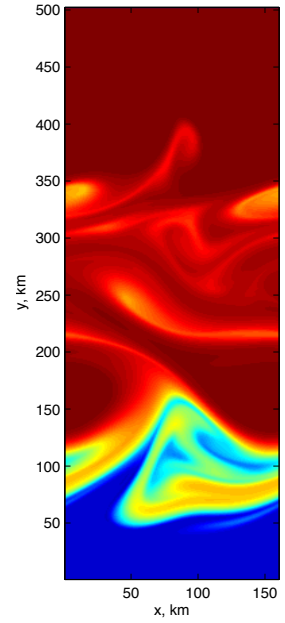

(a) day $200 \nu_{h}=200$

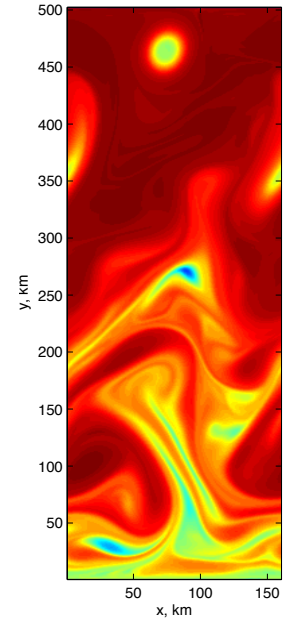

(b) day $200 \nu_{h}=20$

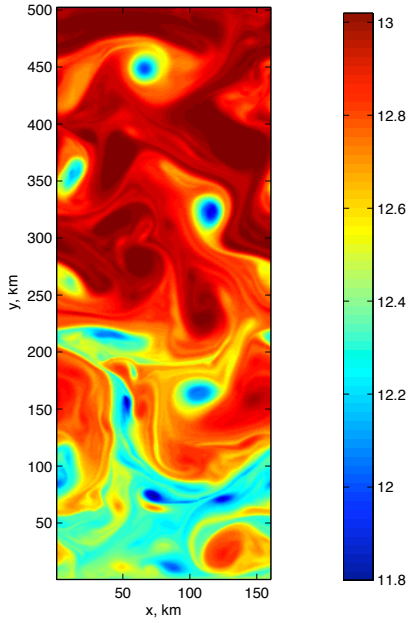

(c) day $200 \nu_{h}=1$

Figure 10: Baroclinic eddies test case: POP model, showing temperature, as in Figure 9, for day 200. Simulations were conducted with both z-level and z-star. Shown are z-level in (a) and z-star in (b, c)

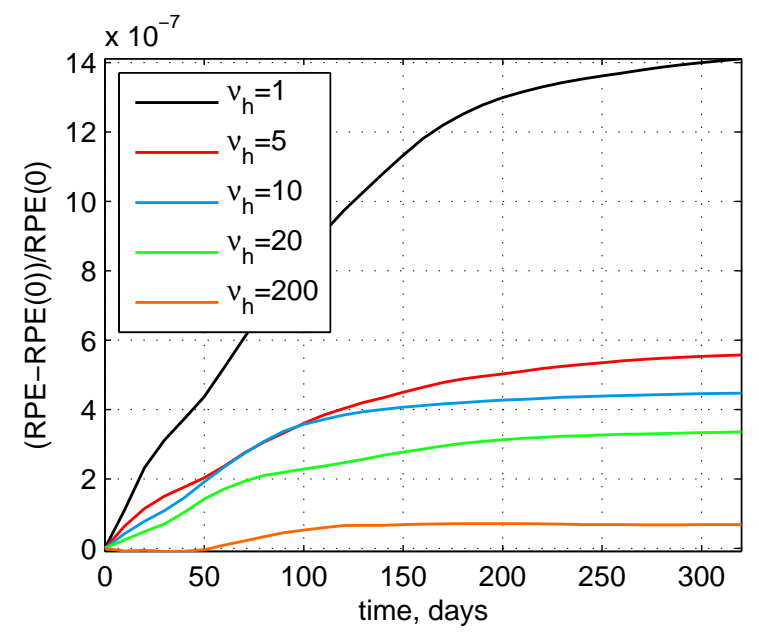

Figure 11: Baroclinic eddies test case: Normalized RPE for the $4 \mathrm{~km}$ using MPAS-Ocean with z-star on a hexagon grid. Horizontal viscosity values of $\nu_{h}=1,5,10,20$, and 200 are associated with grid Reynolds numbers of $R e_{\Delta}=350,70,35,15$, and 1 . 

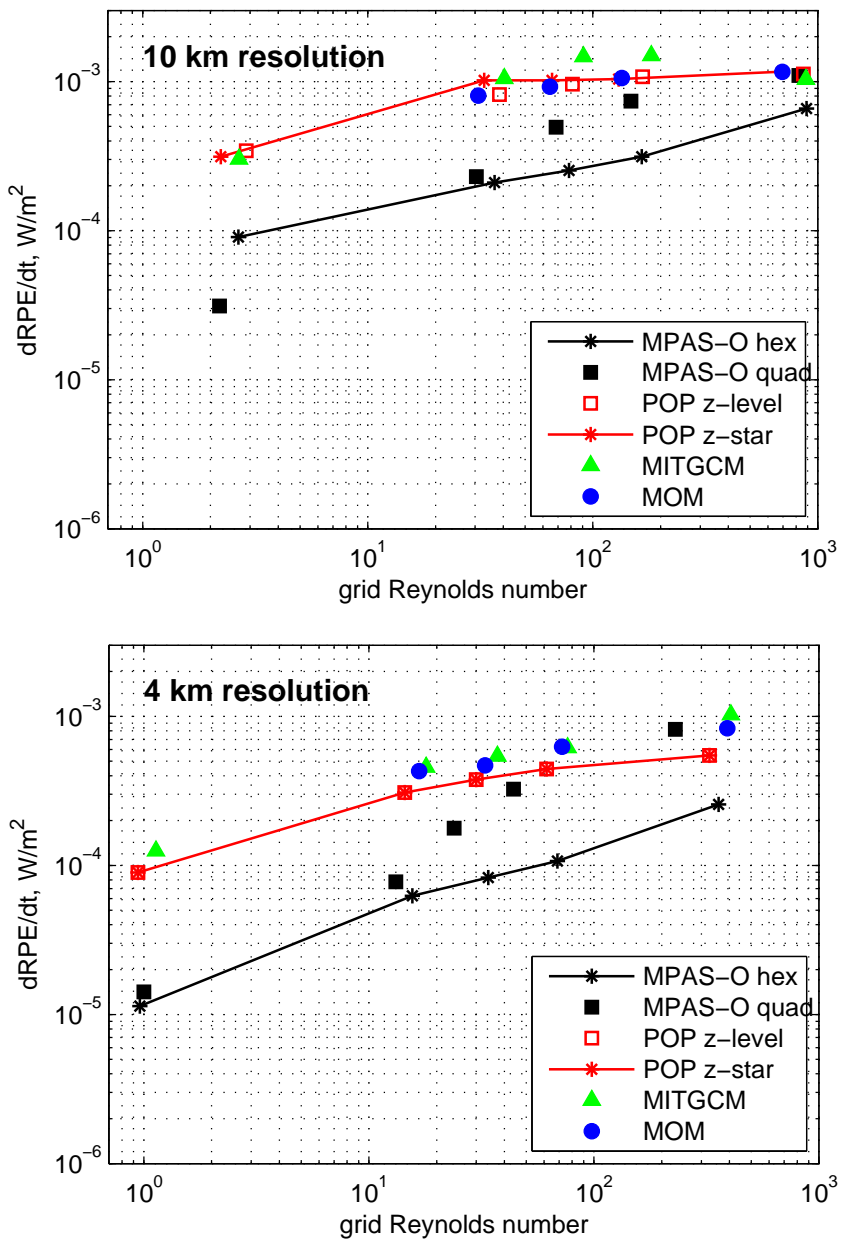

(b)

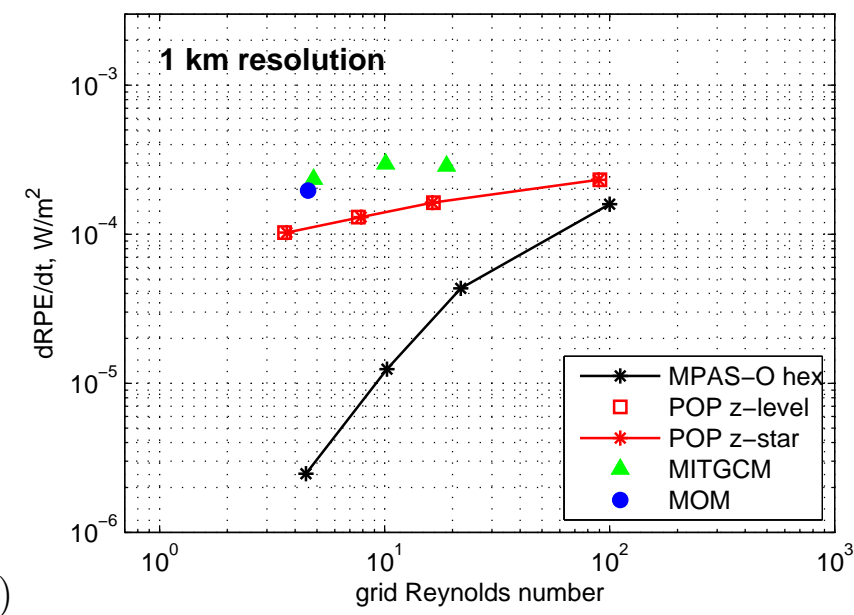

Figure 12: Baroclinic eddies test case: Rate of change of RPE using the $\nu_{h}$ values in Figure 11 for grid cell resolutions of (a) $104 \mathrm{kn}$, (b) $4 \mathrm{~km}$, and (c) $1 \mathrm{~km}$. Derivative data is averaged from day 1-320 for POP and MPAS-Ocean and 3-319 days for MITgcm and MOM. MPAS-Ocean was tested with regularly spaced hexagon (hex) and quadrilateral (quad) horizontal grids, both using z-star. MPAS-Ocean z-level simulations overlay z-star data and are not shown. 


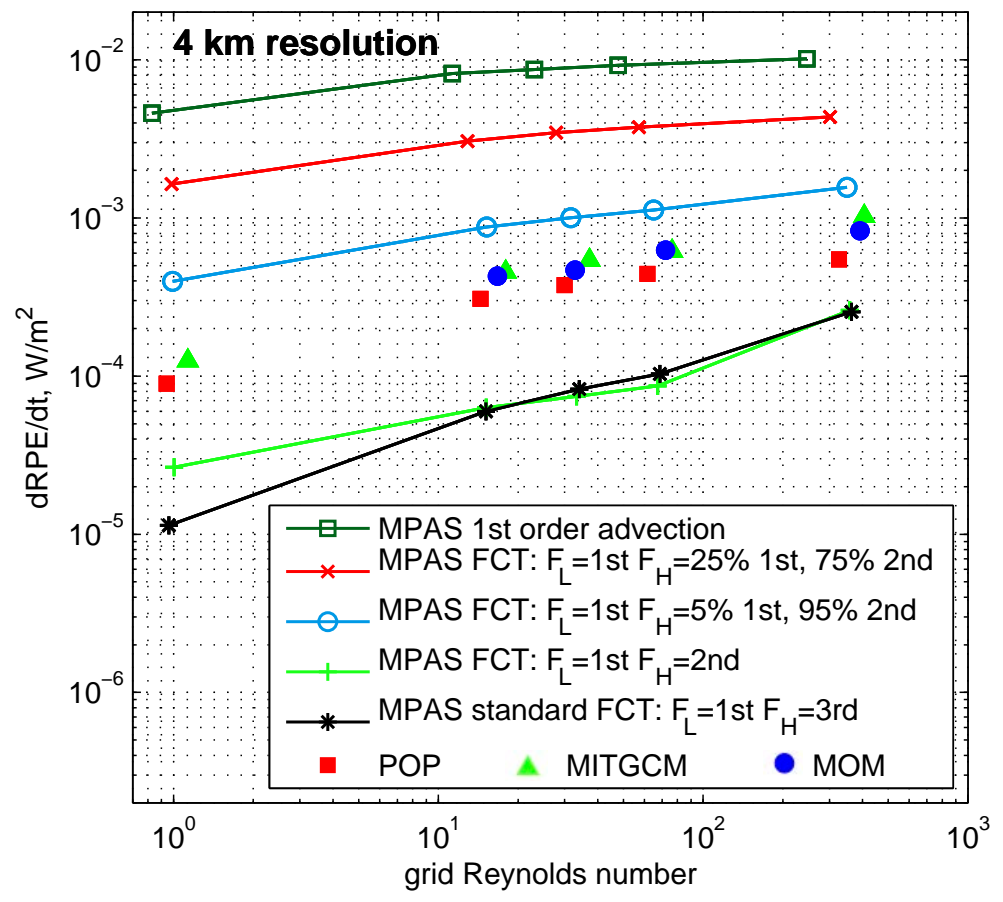

Figure 13: Baroclinic eddies test case: Comparison of mixing by varying the high-order flux component $\left(F_{H}\right)$ within the flux-corrected transport (FCT) scheme in MPAS-Ocean with hexagon cells. The low-order flux component $\left(F_{L}\right)$ is always first-order upwind advection. As the order of $F_{H}$ is increased, $d R P E / d t$ decreases, up to the standard FCT scheme, which produces the least mixing. Other model data and timing is identical to Figure 12b. 


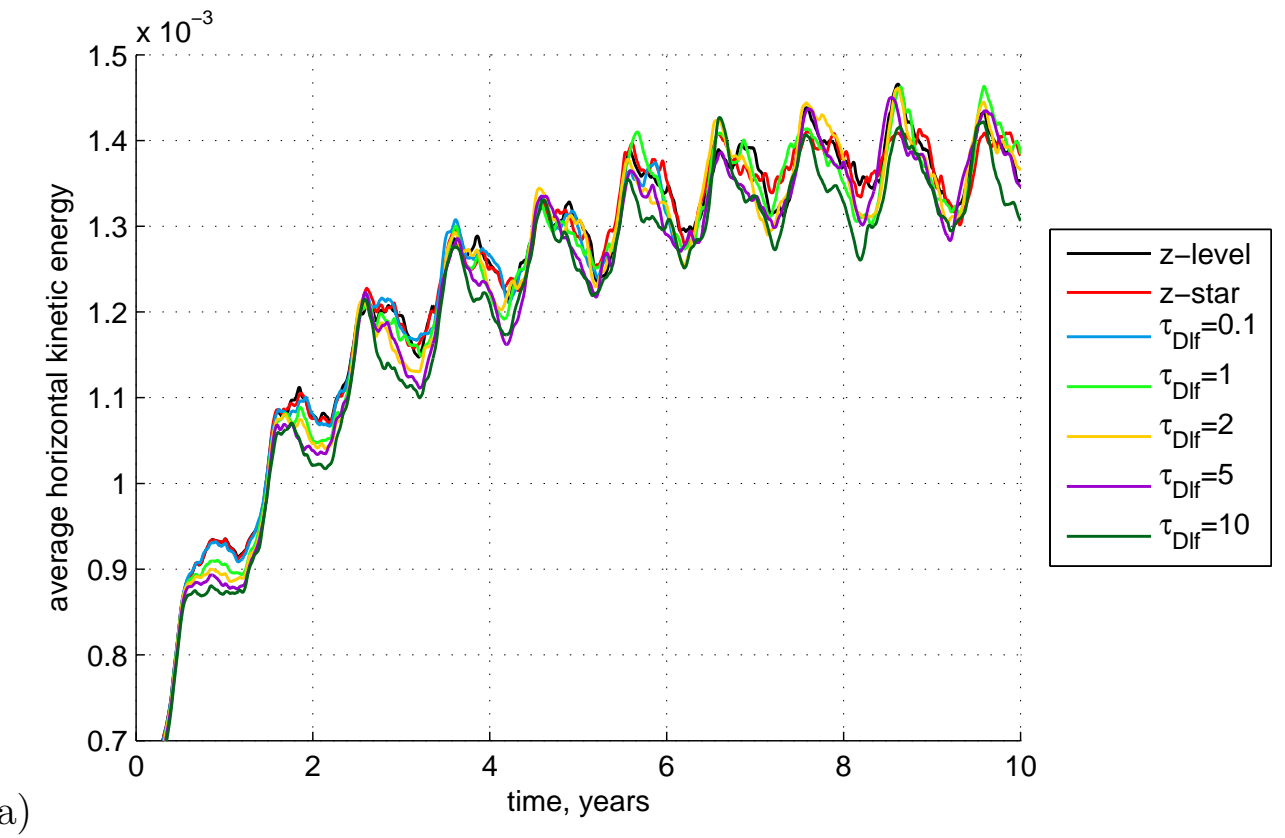

(a)

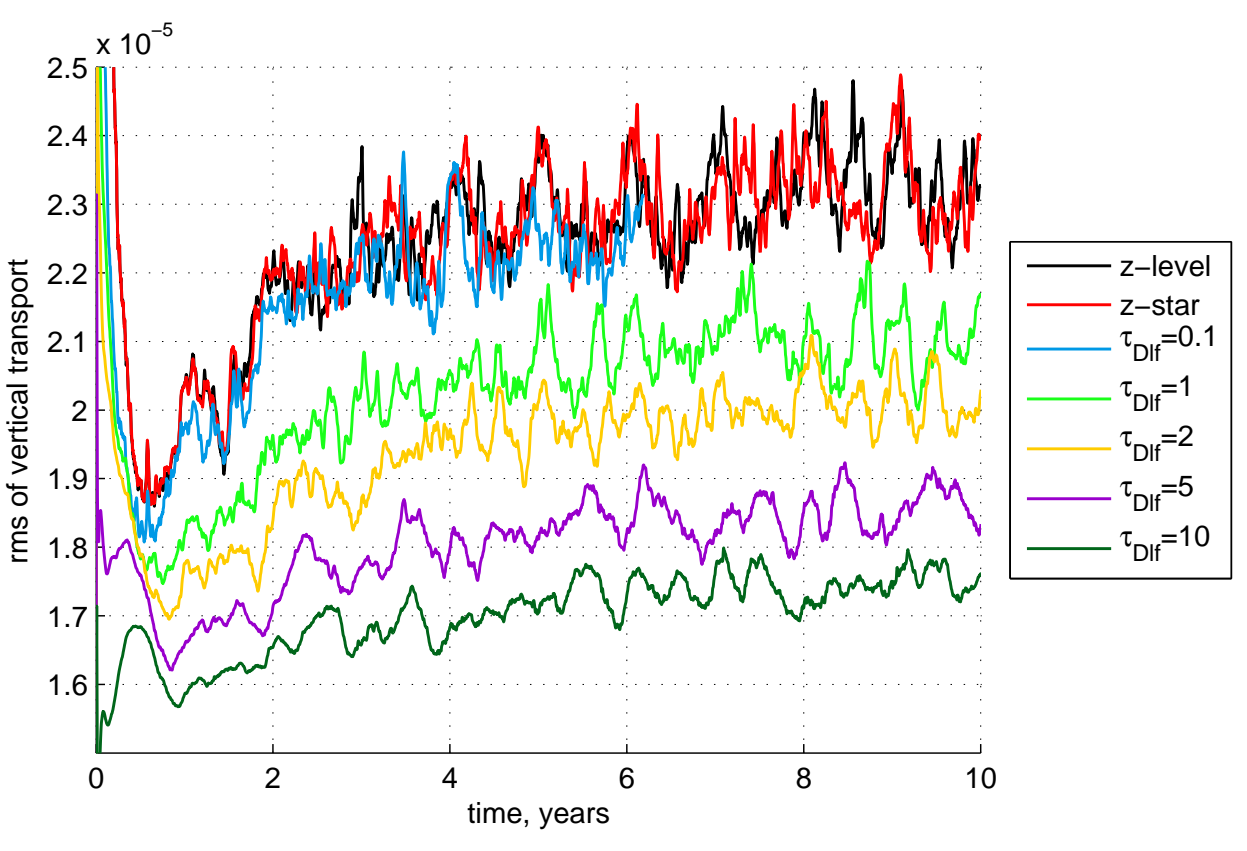

Figure 14: Global spin-up case: domain-average horizontal kinetic energy, $\mathrm{m}^{2} \mathrm{~s}^{-2}$ (a) and vertical cross-coordinate transport, $\mathrm{m} / \mathrm{s}$ (b) for the global $60 \mathrm{~km}$ spin-up simulations using MPAS-Ocean, with varying values of the filter time scale $\left(\tau_{D l f}\right)$. Legend entries with $\tau_{D l f}$ indicates that z-tilde was used in addition to the z-star vertical coordinate. 

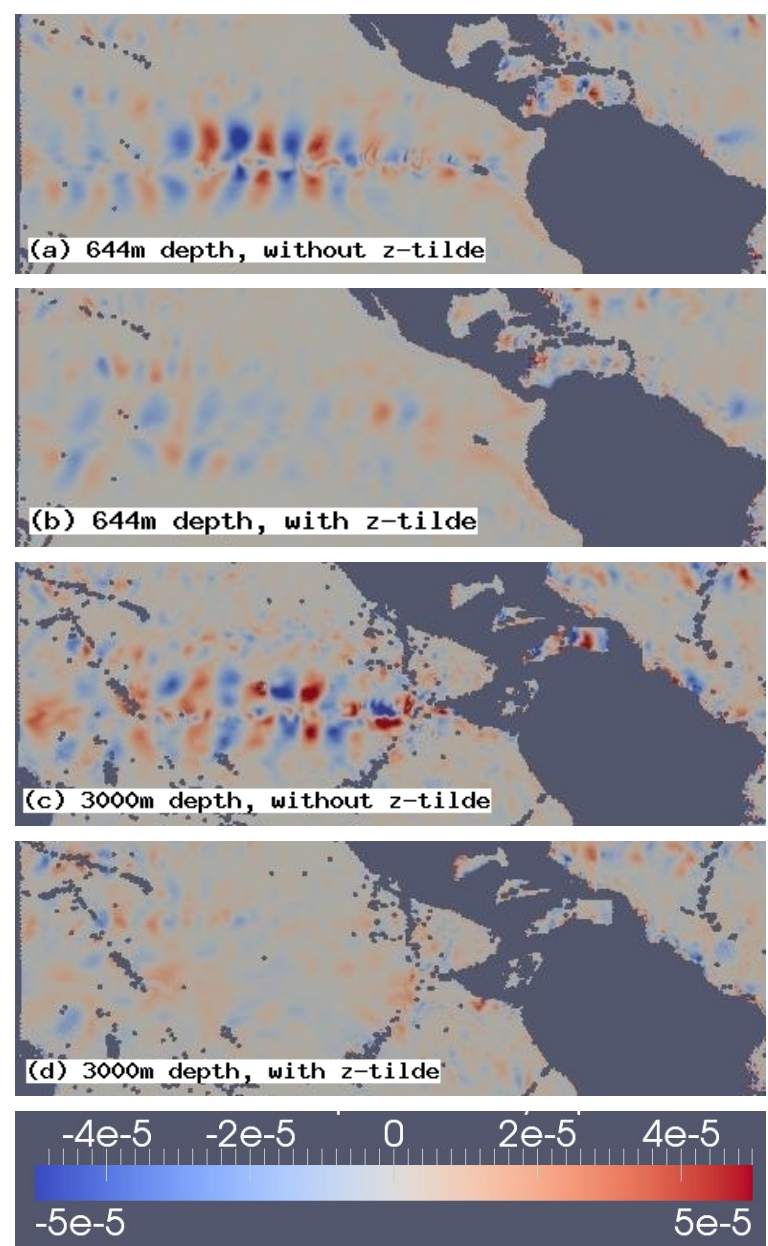

Figure 15: Global spin-up case: z-tilde significantly reduces vertical cross-coordinate transport $(\mathrm{m} / \mathrm{s})$. Images are at 9.5 years and a depth of $644 \mathrm{~m}(\mathrm{a}, \mathrm{b})$ and $3000 \mathrm{~m}(\mathrm{c}, \mathrm{d})$ using a z-star vertical coordinate without z-tilde (a, c) and with z-tilde and $\tau_{D l f}=5$ days (b, d). Images show equatorial Pacific, extending from $20 \mathrm{~S}$ to $30 \mathrm{~N}$ latitude and $180 \mathrm{~W}$ to $35 \mathrm{~W}$ longitude. All simulations have a grid resolution of $60 \mathrm{~km}$. 

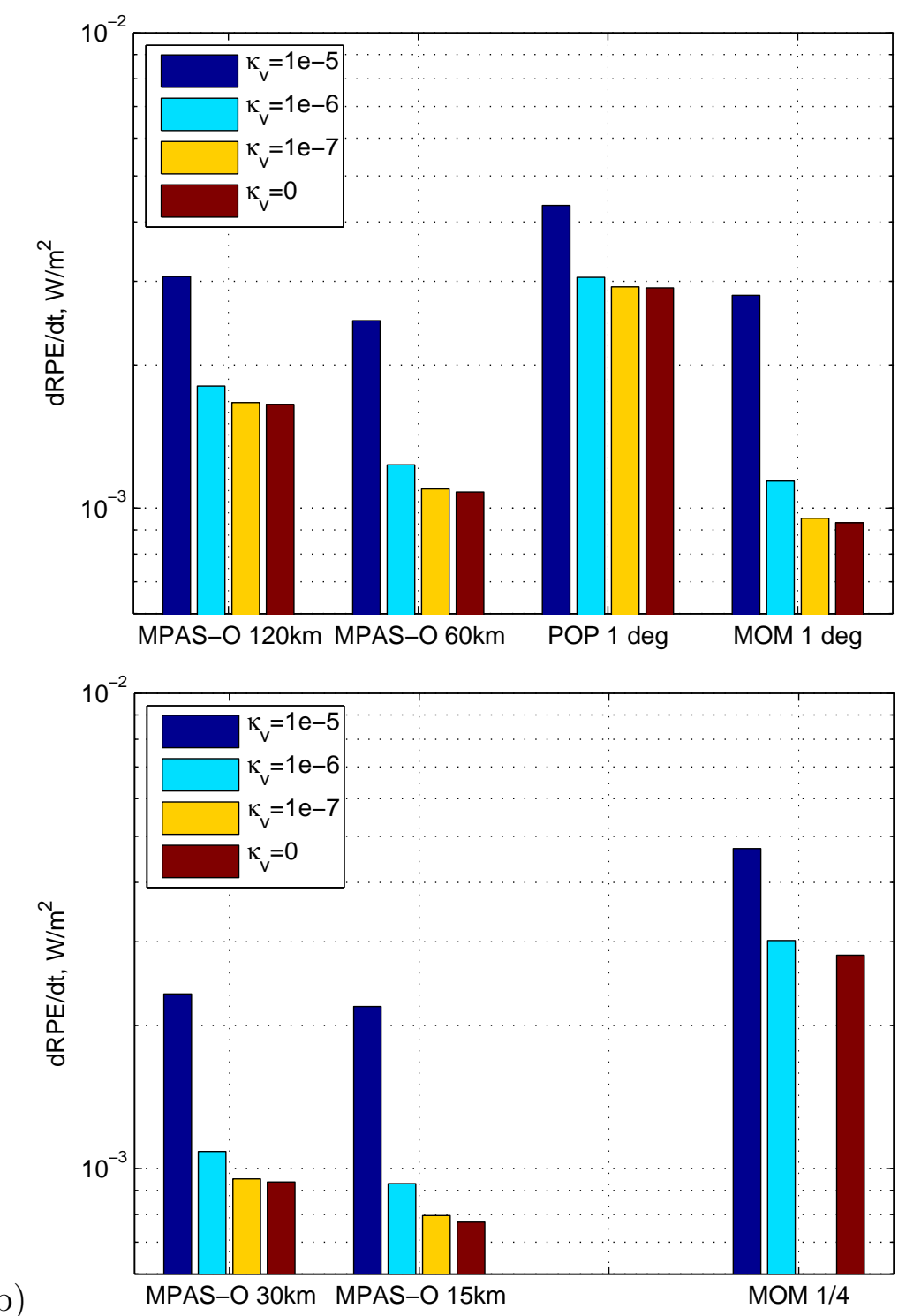

Figure 16: Global spin-down case: Rate of change of RPE for (a) lower and (b) higher resolutions, averaged over day 20 to 70 . MPAS-Ocean cases use a z-star vertical coordinate (no cases use z-tilde). Grid Reynolds numbers are 5, 7.5, 8.5, and 9 for the $120 \mathrm{~km}, 60$ $\mathrm{km}, 30 \mathrm{~km}$, and $15 \mathrm{~km}$ resolutions, respectively, when $\kappa_{v}=0$. 


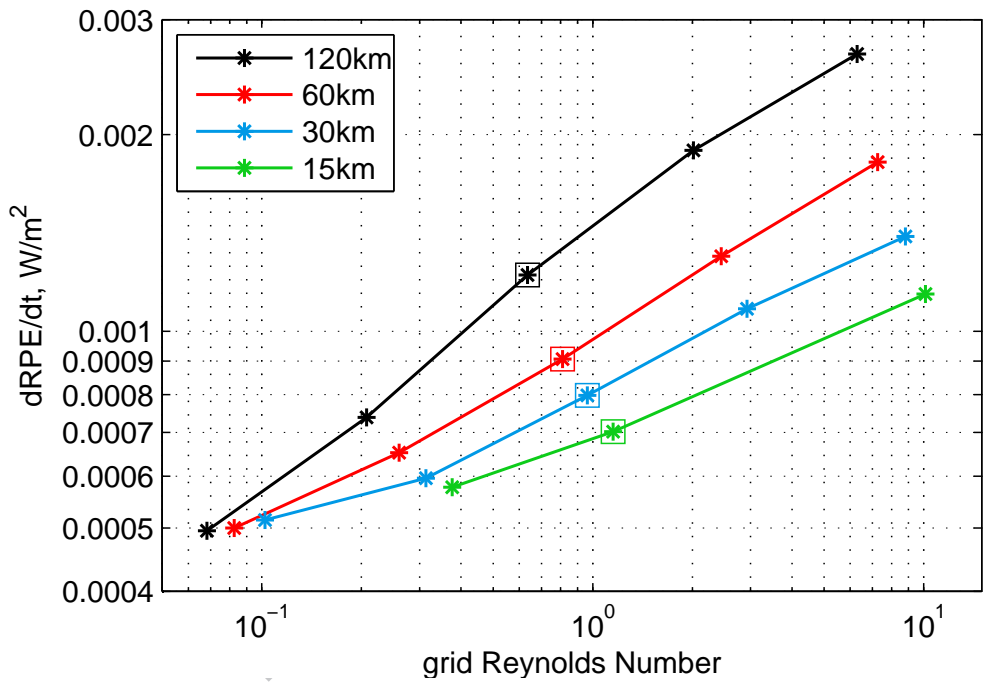

Figure 17: Global spin-down case: Rate of change of RPE versus grid Reynolds number for MPAS-Ocean simulations with zero vertical diffusion, averaged over day 20 to 70 , using a z-star vertical coordinate. No cases use z-tilde. For a fixed resolution, the grid Reynolds number varies with the biharmonic viscosity coefficient. Squares highlight standard values of grid Reynolds numbers for MPAS-Ocean simulations, which are near one. 